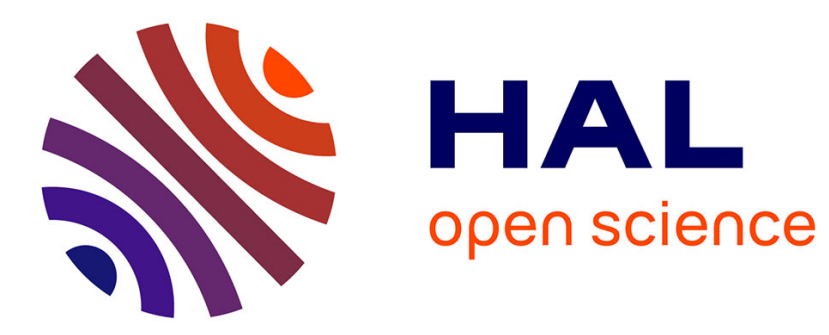

\title{
Mechanism of Spin-Exchange Internal Conversion: Practical Proxies for Diabatic and Nonadiabatic Couplings
}

\author{
Shuming Bai, Mario Barbatti
}

\section{- To cite this version:}

Shuming Bai, Mario Barbatti. Mechanism of Spin-Exchange Internal Conversion: Practical Proxies for Diabatic and Nonadiabatic Couplings. Journal of Chemical Theory and Computation, 2019, 15 (3), pp.1503-1513. 10.1021/acs.jctc.8b00923 . hal-02288617

\section{HAL Id: hal-02288617 https://hal-amu.archives-ouvertes.fr/hal-02288617}

Submitted on 15 Sep 2019

HAL is a multi-disciplinary open access archive for the deposit and dissemination of scientific research documents, whether they are published or not. The documents may come from teaching and research institutions in France or abroad, or from public or private research centers.
L'archive ouverte pluridisciplinaire HAL, est destinée au dépôt et à la diffusion de documents scientifiques de niveau recherche, publiés ou non, émanant des établissements d'enseignement et de recherche français ou étrangers, des laboratoires publics ou privés. 


\title{
Mechanism of Spin-Exchange Internal Conversion: \\ Practical Proxies for Diabatic and
}

Nonadiabatic Couplings

\author{
Shuming Bai* and Mario Barbatti*
}

Aix Marseille Univ, CNRS, ICR, Marseille, France

\begin{abstract}
Spin-exchange internal conversion (SEIC) is a general class of reactions having singlet fission and triplet fusion as particular cases. Based on a charge-transfer (CT)-mediated mechanism and analytical derivation with a model Hamiltonian, we propose proxies for estimating the coupling strength in both diabatic and adiabatic pictures for general SEIC reactions. In the diabatic picture, we demonstrated the existence of a bilinear relationship between the coupling strength and molecular orbital overlap, which provides a practical way to predict diabatic couplings. In the adiabatic picture, we showed that nonadiabatic couplings can be approximated by simple functions of the wave function CT coefficients. These approaches were verified through the investigation of singlet oxygen photosensitization, where both ${ }^{1} \Delta_{\mathrm{g}}$ and ${ }^{1} \Sigma_{\mathrm{g}}$ oxygen states can be competitively generated by a triplet fusion reaction. The interplay between the CT-mediated mechanism, the spatial factors of the bimolecular complex, and the electronic structure of the oxygen molecule during the reaction explains the curiously small coupling to the ${ }^{1} \Sigma_{\mathrm{g}}$ state along specific incidence directions. The results from both the diabatic and adiabatic pictures provide a comprehensive understanding of the reaction mechanism, which applies to general SEIC problems.
\end{abstract}




\section{INTRODUCTION}

Spin-exchange internal conversion (SEIC) forms a class of reactions where the global spin of a molecular assembly is conserved, while the spin of the monomers is changed. The most famous example of such a reaction is the singlet fission (SF) ${ }^{1-4}$ following the general formula

$$
{ }^{1}\left[{ }^{1} \mathrm{~A}+{ }^{1} \mathrm{~B}\right] \rightarrow{ }^{1}\left[{ }^{3} \mathrm{~A}+{ }^{3} \mathrm{~B}\right]
$$

which has been under scrutiny recently due to its potential for enhancing the efficiency of organic photovoltaics (usually A and B are the same molecule). ${ }^{5,6}$ Another example of SEIC is the internal-conversion triplet fusion reaction $(\mathrm{TF})$

$$
{ }^{1}\left[{ }^{3} \mathrm{~A}+{ }^{3} \mathrm{~B}\right] \rightarrow{ }^{1}\left[{ }^{1} \mathrm{~A}+{ }^{1} \mathrm{~B}\right] .
$$

This reaction yielding a singlet complex is one among nine possible outcomes, the other being five quintet complexes and three triplet complexes. Reaction (2) is expected to dominate the fusion rate because of internal conversion tends to be faster than intersystem crossing. ${ }^{7,8}$ This reaction was recently applied on functional molecular devices as the formally inverse reaction of singlet fission. ${ }^{9,10}$ It is also at the core of singlet oxygen photosensitization, ${ }^{11-14}$ which happens through

$$
{ }^{1}\left[{ }^{3} \mathrm{PS}+{ }^{3} \mathrm{O}_{2}\right] \rightarrow{ }^{1}\left[{ }^{1} \mathrm{PS}+{ }^{1} \mathrm{O}_{2}\left({ }^{1} \Sigma \text { or }{ }^{1} \Delta\right)\right]
$$

where PS is a photosensitizer.

Theoretical and computational chemistry have contributed to the study of SEIC reactions since the early $1960 \mathrm{~s}^{15,16}$ and many recent advances in both fronts, $\mathrm{SF}^{5,17-19}$ and $\mathrm{TF},{ }^{12,20}$ have brought new insights on the molecular mechanism and the reaction rate calculation. As usual in any unimolecular reaction, both the energy of the involved states and the coupling between them should be considered to estimate reaction rates. ${ }^{1}$ State energies can be routinely calculated with quantum chemical methods, either to the entire complex or considering each monomer individually. ${ }^{6,21,22}$ However, the calculation of the coupling strength for SEIC reactions - usually an intermolecular energy transfer processes in weak-coupling regime - is still a challenging task, especially considering that systems of interest may correspond to large 
molecules in floppy organic crystals. For instance, in the search for the optimal way to treat these couplings, topics like the role of charge transfer (CT) configurations and the choice between diabatic and adiabatic pictures have been intensely discussed. ${ }^{2,4,18,23,24}$

The diabatic picture has been a popular option because the diabatic coupling can be directly applied in the rate models such as the Fermi's golden rule. ${ }^{117}$ Many different approaches to estimate the diabatic coupling have been proposed mainly based on two strategies. The first strategy is the direct calculation from predefined diabatic wave functions, ${ }^{25}$ such as in the constrained density functional theory with configuration interaction (CDFT-CI) method, using the constraint of charge/spin density. ${ }^{17,26}$ Direct calculation holds a clear physical meaning, but it may miss intermolecular electronic correlations. ${ }^{2}$ The second strategy is diabatization based on the calculation of adiabatic states, e.g., with the fragment spin difference method, ${ }^{19}$ and the N/D method based on nonadiabatic couplings (NAC) and differences of energy gradients. ${ }^{27}$ Although globally applicable diabatization is still a challenging subject in theoretical chemistry ${ }^{28,}$ ${ }^{29}$ various effective diabatization approaches have been developed, ${ }^{30,} 31$ especially with recent breakthroughs for relative large molecular systems. ${ }^{32,33}$

The adiabatic picture has also been widely applied to study SEIC. ${ }^{2,}{ }^{18}$ Nonadiabatic couplings can be directly computed based on the wave function of the whole molecular complex, with the intermolecular correlation automatically considered. Nevertheless, the adiabatic picture also has two obvious disadvantages. First, NAC is not connected to the reaction rate as conveniently as the diabatic coupling ${ }^{34}$ (although it is directly used in nonadiabatic mixed quantum-classical dynamics simulation ${ }^{35}$ ). Second, NAC is not a quantity readily available for most of quantum chemical levels. ${ }^{36}$ Such practical difficulty to calculate NACs has led to a search for suitable proxies to replace them. For instance, researching an SF reaction, Morrison and Herbert took the direct product of monomeric states as the wave function basis within the ab initio Frenkel-Davydov exciton model, ${ }^{37}$ to calculate the adiabatic state and NAC, instead 
of the direct calculation of the entire dimer system. ${ }^{38}$ In another study, Feng, Luzanov, and Krylov proposed a proxy for NAC based on the reduced one-particle transition between initial and final adiabatic states and used it to evaluate the NAC strength variation with different intermolecular arrangements. ${ }^{18}$

In a recent series of research works, we have focused on the TF reaction given in eq (3), the photosensitization reaction yielding singlet oxygen. ${ }^{11}{ }^{12}$ To deal with weakly coupled floppy complexes, we proposed the Divide-to-Conquer (DtC) model for reaction rates in ref ${ }^{11}$ (for convenience, it is also briefly discussed in the Supporting Information, SI). In the DtC model both diabatic and nonadiabatic couplings are available for the same reaction coordinates, and they are directly correlated to each other by a simple function. This feature provides us with an excellent opportunity to clarify the relationship between adiabatic and diabatic pictures in SEIC reactions. Moreover, to work with the heterodimer PS-O $\mathrm{O}_{2}$ (rather than with homodimers usually adopted in this type of investigation) also enriches the prototypical range of SEIC research.

Our goal in this work is to derive proxies for diabatic and nonadiabatic couplings for SEIC reactions. These proxies should play a double role of 1) allowing a straightforward estimate of couplings for large systems and 2) providing a direct interpretation of the reaction mechanisms. These proxies will be discussed and validated against quantum-chemical results from our previous investigations on the $\mathrm{PS}-\mathrm{O}_{2}$ complex.

\section{THEORETICAL FRAMEWORK}

\subsection{Diabatic Picture}

In the diabatic picture, we consider the reaction with several different diabatic states: the initial state $\psi_{\text {ini }}$, the final state $\psi_{f i n}$, and the intermediate CT state $\psi_{C T}{ }^{1,24}$ To build a general picture of the SEIC reaction, we start with an $\mathbf{A B}$ complex consisting of molecules $\mathbf{A}$ and $\mathbf{B}$, where $\mathbf{A}$ and $\mathbf{B}$ can be identical or not. For SEIC reaction, the Förster interaction is spin forbidden, ${ }^{39}$ while Dexter interaction is necessary, ${ }^{40}$ and the diabatic coupling between the states arises from two types of interaction paths: a direct interaction 
and a CT-mediated superexchange interaction. ${ }^{1}$ As an example, the interaction pathways of one special case belonging to the TF reaction are visualized in Figure 1.

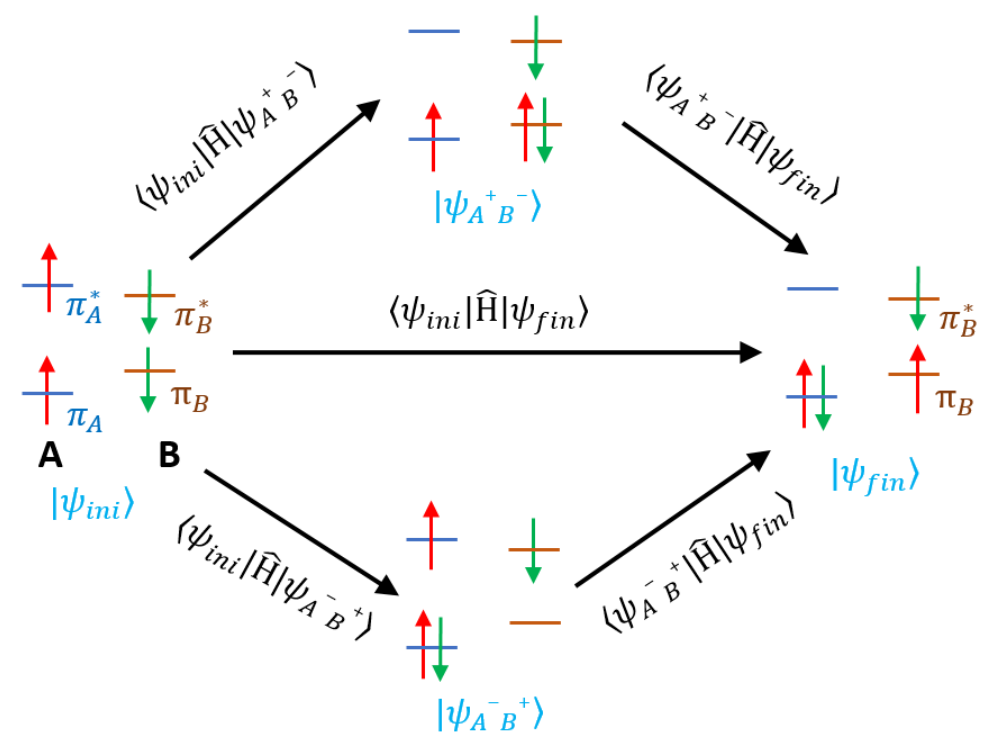

Figure 1. Interaction paths for one an example of TF reaction in the $\mathbf{A B}$ complex. Top and bottom pathways indicate CT-mediated mechanisms. The pathway in the middle is the direct mechanism.

With these two CT states as the mediate, the total diabatic coupling is given by ${ }^{1,39,41}$

$$
V_{S E I C} \approx \sum_{i=1}^{2} \frac{\left\langle\psi_{i n i}|\hat{H}| \psi_{C T_{i}}\right\rangle\left\langle\psi_{C T_{i}}|\hat{H}| \psi_{f i n}\right\rangle}{E_{C T_{i}}}-\left\langle\psi_{i n i}|\hat{H}| \psi_{f i n}\right\rangle
$$

where $C T_{1}$ and $C T_{2}$ correspond to the two $C T$ states $\left(\mathbf{A}^{-} \mathbf{B}^{+}\right.$and $\mathbf{A}^{+} \mathbf{B}^{-}$, as shown in Figure 1), which can connect the initial and final states through one-electron transfer paths. $\hat{H}$ is the electronic Hamiltonian operator, and $E_{C T_{i}}$ is the energy of the CT state $i$ taking the initial state energy as the reference. From the perspective of A, these two interaction pathways are i) first-donate-then-accept-electron (top) and ii) firstaccept-then-donate-electron (bottom). In eq (4), the first term in the right side corresponds to coupling through the CT-mediated mechanisms, and the second term is the coupling through the direct mechanism. 
Assuming that the quantum interstate electronic coupling is based on the orbital overlap, a linear relationship between the electronic coupling and the overlap of the corresponding diabatic state wave functions was proposed to evaluate electron transfer coupling approximately, ${ }^{42}$ which has been confirmed in various works. ${ }^{43-45}$ In Ref. ${ }^{43}$ they have tested the simple expression

$$
\Delta_{a C T}=\left\langle\psi_{a}|\hat{H}| \psi_{C T}\right\rangle=C\left\langle\psi_{a} \mid \psi_{C T}\right\rangle \approx C\left\langle\phi_{a} \mid \phi_{C T}\right\rangle
$$

where $\Delta_{a C T}$ is the electronic coupling between the initial state $\left(\psi_{a}\right)$ and charge transfer $\left(\psi_{C T}\right)$ states, and $C$ is a constant. $\left\langle\psi_{a} \mid \psi_{C T}\right\rangle$ is the overlap between the two wave functions, and it is approximated by $\left\langle\phi_{a} \mid \phi_{C T}\right\rangle$, the overlap between the two frontier molecular orbitals (MOs), $\phi_{a}$ and $\phi_{C T}$. Because of the electron transfer nature, $\phi_{a}$ and $\phi_{C T}$ are mainly located at the donor and acceptor molecules, respectively. The linear relationship works very well for a very diverse set of donor-acceptor pairs in different random orientations and distances, sharing the same constant $C .{ }^{43}$ For simplicity, we assume the term $\mathrm{C}$ as the same constant. (Although even using different $\mathrm{C}$ for different coupling terms, the conclusion will not change.) $C$ was reported to lie between 3 and $15 \mathrm{eV}$ in different studies, ${ }^{42,43,45}$ and the variation may be due to the accuracy of the overlap calculation.

The coupling terms for the CT-mediated mechanism in eq (4) are exactly the charge-transfer coupling appearing in eq (5). Therefore, we can rewrite the CT-mediated diabatic coupling in terms of the overlap of molecular orbitals

$$
\begin{aligned}
\sum_{i=1}^{2} \frac{\left\langle\psi_{i n i}|\hat{H}| \psi_{C T_{i}}\right\rangle\left\langle\psi_{C T_{i}}|\hat{H}| \psi_{f i n}\right\rangle}{E_{C T_{i}}} & =C^{2}\left(\frac{1}{E_{A^{+} B^{-}}}+\frac{1}{E_{A^{-} B^{+}}}\right)\left\langle\phi_{A} \mid \phi_{B}\right\rangle\left\langle\phi_{B}^{\prime} \mid \phi_{A}^{\prime}\right\rangle \\
& =\eta\left\langle\phi_{A} \mid \phi_{B}\right\rangle\left\langle\phi_{B}^{\prime} \mid \phi_{A}^{\prime}\right\rangle
\end{aligned}
$$

which is simpler to be analyzed. In this equation, $\eta=C^{2}\left(E_{A^{+} B^{-}}^{-1}+E_{A^{-} B^{+}}^{-1}\right)$. 
As shown in eq (6), the two CT-mediated pathways give the same orbital overlap terms, because the frontier molecular orbitals involved in the two paths are exactly the opposite. For example, the overlap terms for the two CT-mediated pathways in Figure 1 are $\left\langle\pi_{A}^{*} \mid \pi_{B}\right\rangle\left\langle\pi_{B} \mid \pi_{A}\right\rangle$ and $\left\langle\pi_{B} \mid \pi_{A}\right\rangle\left\langle\pi_{A}^{*} \mid \pi_{B}\right\rangle$, which are the same after a simple reordering. Therefore, with the determined initial and final states, only one total orbital overlap term will regulate the diabatic coupling through CT-mediated mechanism. To predict the magnitude of this term, up to only four MOs $\left(\phi_{A}, \phi_{B}, \phi_{A}^{\prime}, \phi_{B}^{\prime}\right)$ are necessary to calculate it. (Four MOs are needed if $\phi_{A} \neq \phi_{A}^{\prime}$ and $\phi_{B} \neq \phi_{B}^{\prime}$, as shown in Figure S3 of SI.)

The last term in eq (4), corresponding to the direct coupling, is usually smaller than the CT-mediated terms, if the CT state energy is not too high. ${ }^{1,22}$ It comes from the two-electron exchange integral, and we can simplify it with a two-electron/two-orbital picture as $\left\langle\psi_{\text {ini }}|\mathbf{M}| \psi_{\text {fin }}\right\rangle=\int d r_{1} d r_{2} \phi_{A}\left(r_{1}\right) \phi_{B}\left(r_{1}\right) \frac{1}{\left|r_{1}-r_{2}\right|} \phi_{B}^{\prime}\left(r_{2}\right) \phi_{A}^{\prime}\left(r_{2}\right)$. As we can see from this expression, this term is also related to the orbital overlap between the same four frontier MOs as before. If we assume that the orbital overlap of $\left(\phi_{A}, \phi_{B}\right)$ and $\left(\phi_{A}^{\prime}, \phi_{B}^{\prime}\right)$ are distributed differently in the space (for most cases it is true), then we can find a fixed or averaged $d_{x}=\left|\bar{r}_{1}-\bar{r}_{2}\right|$ and integrate the two overlap parts separately

$$
\begin{aligned}
\left\langle\psi_{\text {ini }}|\mathbf{H}| \psi_{f i n}\right\rangle & =\int d r_{1} d r_{2} \phi_{A}\left(r_{1}\right) \phi_{B}\left(r_{1}\right) \frac{1}{\left|r_{1}-r_{2}\right|} \phi_{B}^{\prime}\left(r_{2}\right) \phi_{A}^{\prime}\left(r_{2}\right) \\
& \approx \frac{1}{d_{x}} \int d r_{1} \phi_{A}\left(r_{1}\right) \phi_{B}\left(r_{1}\right) \int d r_{2} \phi_{B}^{\prime}\left(r_{2}\right) \phi_{A}^{\prime}\left(r_{2}\right) \\
& =\frac{1}{d_{x}}\left\langle\phi_{A} \mid \phi_{B}\right\rangle\left\langle\phi_{B}^{\prime} \mid \phi_{A}^{\prime}\right\rangle
\end{aligned}
$$

Although this is a rough approximation, considering the tiny contribution of direct coupling compared to the CT-mediate coupling, this treatment still provides an effective way to include any direct coupling 
effect. The term $d_{x}$ should be mainly determined by the orbital shape and the distance between $\mathbf{A}$ and $\mathbf{B}$. Combing eqs (4), (6) and (7), now we have the expression of diabatic coupling from the orbital overlap:

$$
\begin{aligned}
V_{\text {SEIC }} & =\eta\left\langle\phi_{A} \mid \phi_{B}\right\rangle\left\langle\phi_{B}^{\prime} \mid \phi_{A}^{\prime}\right\rangle+\frac{1}{d_{x}}\left\langle\phi_{A} \mid \phi_{B}\right\rangle\left\langle\phi_{B}^{\prime} \mid \phi_{A}^{\prime}\right\rangle \\
& =\left(\eta+\frac{1}{d_{x}}\right)\left\langle\phi_{A} \mid \phi_{B}\right\rangle\left\langle\phi_{B}^{\prime} \mid \phi_{A}^{\prime}\right\rangle
\end{aligned}
$$

which can be finally written as

$$
V_{S E I C}=\eta_{\text {total }}\left\langle\phi_{A} \mid \phi_{B}\right\rangle\left\langle\phi_{B}^{\prime} \mid \phi_{A}^{\prime}\right\rangle
$$

$\eta_{\text {total }}=\eta+d_{x}^{-1}$ includes the effects from both CT-mediate mechanism and direct mechanism $\left(\eta ? d_{x}^{-1}\right)$. In

this work, we do not compute $\eta_{\text {total }}$ and exclusively focus on the essential role of the total overlap term to qualitatively explain certain features of the $\mathrm{PS}-\mathrm{O}_{2}$ SEIC reaction.

As a conclusion of this part, we utilized the bilinear relationship between electron transfer coupling and frontier MO overlaps, ${ }^{43}$ to derive a clear relationship between the final diabatic coupling and the total orbital overlap as a product of two MO overlap terms. This relationship can be applied to predict the diabatic coupling strength, especially its variation in intermolecular space.

We note here that Feng and Krylov also tested the effect of orbital overlap on the coupling strength, and found only a weak correlation between them. ${ }^{46}$ However, they considered the NAC strength, while our derivation in this sections is for the diabatic coupling. Moreover, with our derivation, we found a direct relation between the diabatic coupling an the product of orbital overlaps (eq (8)), while in their work they took the sum of the orbital overlaps instead.

\subsection{Adiabatic Picture}

In the adiabatic picture, the transition between adiabatic states is mediated by the nonadiabatic coupling vector 


$$
\mathbf{F}_{I J} \equiv\left\langle\Psi_{I} \mid \frac{\partial \Psi_{J}}{\partial \boldsymbol{R}}\right\rangle
$$

where $\mathbf{R}$ is the nuclear coordinate. To derive a proxy for NAC specially adapted for SEIC reactions, we start from the diabatic initial state $\psi_{i n i}$, final state $\psi_{\text {fin }}$, and the intermediate CT state $\psi_{C T}$ as mentioned in subsection 2.1. The adiabatic wave functions are obtained by diagonalizing the following system Hamiltonian

$$
\mathbf{H}_{\text {SEIC }}=\left(\begin{array}{ccc}
E_{\mathrm{ini}}=0 & 0 & \Delta_{i C T} \\
0 & E_{f i n} & \Delta_{f C T} \\
\Delta_{i C T} & \Delta_{f C T} & E_{C T}
\end{array}\right)
$$

For SEIC reaction, usually the CT state energy is higher than the other two states, ${ }^{38,47,48}$ and the diabatic coupling strength for intermolecular $\mathrm{CT}$ is limited, so we have the following relationship: $\left|E_{C T}-E_{i n i}\right|>\left|E_{C T}-E_{f i n}\right| ? \Delta_{i C T}$ or $\Delta_{f C T}$. Then, the adiabatic wave functions are

$$
\begin{aligned}
\Psi_{1} & =c_{i n i}^{(1)} \psi_{i n i}+\frac{\Delta_{i C T} \Delta_{f C T}}{E_{f i n} E_{C T}} \psi_{f i n}-\frac{\Delta_{i C T}}{E_{C T}} \psi_{C T} \\
& =c_{i n i}^{(1)} \psi_{i n i}+c_{f i n}^{(1)} \psi_{f i n}+c_{C T}^{(1)} \psi_{C T} ; \quad\left(E_{1} \approx 0\right) \\
\Psi_{2} & =c_{f i n}^{(2)} \psi_{f i n}-\frac{\Delta_{i C T} \Delta_{f C T}}{E_{f i n}\left(E_{C T}-E_{f i n}\right)} \psi_{i n i}-\frac{\Delta_{f C T}}{E_{C T}-E_{f i n}} \psi_{C T} \\
& =c_{f i n}^{(2)} \psi_{f i n}+c_{i n i}^{(2)} \psi_{i n i}+c_{C T}^{(2)} \psi_{C T} ; \quad\left(E_{2} \approx E_{f i n}\right) \\
\Psi_{3} & =c_{C T}^{(3)} \psi_{C T}+\frac{\Delta_{i C T}}{E_{C T}} \psi_{i n i}+\frac{\Delta_{f C T}}{E_{C T}-E_{f i n}} \psi_{f i n} \quad\left(E_{3} \approx E_{C T}\right) \\
& =c_{C T}^{(3)} \psi_{C T}+c_{i n i}^{(3)} \psi_{i n i}+c_{f i n}^{(3)} \psi_{f i n} ; \quad
\end{aligned}
$$

In these expressions, $c_{i n i}^{(1)}, c_{f i n}^{(2)}$, and $c_{C T}^{(3)}$ are always near to the unity, which reflects that each adiabatic state is dominated by one of the three diabatic states in the weak coupling region, only with some minor components of other two states. $c_{f i n}^{(1)}$, the mixture of $\psi_{\text {fin }}$ to state 1 , and $c_{\text {ini }}^{(2)}$, the mixture of $\psi_{\text {ini }}$ to state 
2, are much smaller than $c_{C T}^{(1)}$ and $c_{C T}^{(2)}$, the mixture of CT to state1 or state 2. These relations hold because the CT state is directly connected to the initial and final states via diabatic couplings, while $\psi_{\text {ini }}$ and $\psi_{\text {fin }}$ are connected by the superexchange effect of the CT state.

We can derive the NAC between state 1 and state 2 according to eqs (10) and (12)

$$
\begin{aligned}
\mathbf{F}_{12} & =\left\langle\Psi_{1} \mid \frac{\partial \Psi_{2}}{\partial \boldsymbol{R}}\right\rangle \\
& =\left\langle c_{i n i}^{(1)} \psi_{i n i}+c_{f i n}^{(1)} \psi_{f i n}+c_{C T}^{(1)} \psi_{C T} \mid \frac{\partial\left(c_{f i n}^{(2)} \psi_{f i n}+c_{i n i}^{(2)} \psi_{i n i}+c_{C T}^{(2)} \psi_{C T}\right)}{\partial \boldsymbol{R}}\right\rangle
\end{aligned}
$$

Because $\psi_{i n i}, \psi_{f i n}$, and $\psi_{C T}$ are all diabatic states, all the terms like $\left\langle\psi_{x} \mid \frac{\partial \psi_{y}}{\partial \boldsymbol{R}}\right\rangle$ are zero and the gradient of wave functions in eq (13) are negligible. Only the terms with the gradient of the coefficients remain

$$
\begin{aligned}
\mathbf{F}_{12}= & \left\langle c_{i n i}^{(1)} \psi_{i n i} \mid \frac{\partial c_{f i n}^{(2)}}{\partial \boldsymbol{R}} \psi_{f i n}\right\rangle+\left\langle c_{f i n}^{(1)} \psi_{f i n} \mid \frac{\partial c_{f i n}^{(2)}}{\partial \boldsymbol{R}} \psi_{f i n}\right\rangle+\left\langle c_{C T}^{(1)} \psi_{C T} \mid \frac{\partial c_{f i n}^{(2)}}{\partial \boldsymbol{R}} \psi_{f i n}\right\rangle \\
& +\left\langle c_{i n i}^{(1)} \psi_{i n i} \mid \frac{\partial c_{i n i}^{(2)}}{\partial \boldsymbol{R}} \psi_{i n i}\right\rangle+\left\langle c_{f i n}^{(1)} \psi_{f i n} \mid \frac{\partial c_{i n i}^{(2)}}{\partial \boldsymbol{R}} \psi_{i n i}\right\rangle+\left\langle c_{C T}^{(1)} \psi_{C T} \mid \frac{\partial c_{i n i}^{(2)}}{\partial \boldsymbol{R}} \psi_{i n i}\right\rangle \\
& +\left\langle c_{i n i}^{(1)} \psi_{i n i} \mid \frac{\partial c_{\mathrm{CT}}^{(2)}}{\partial \boldsymbol{R}} \psi_{C T}\right\rangle+\left\langle c_{f i n}^{(1)} \psi_{f i n} \mid \frac{\partial c_{C T}^{(2)}}{\partial \boldsymbol{R}} \psi_{C T}\right\rangle+\left\langle c_{C T}^{(1)} \psi_{C T} \mid \frac{\partial c_{C T}^{(2)}}{\partial \boldsymbol{R}} \psi_{C T}\right\rangle \\
= & \frac{\partial c_{f i n}^{(2)}}{\partial \boldsymbol{R}}\left(c_{i n i}^{(1)}\left\langle\psi_{i n i} \mid \psi_{f i n}\right\rangle+c_{f i n}^{(1)}+c_{C T}^{(1)}\left\langle\psi_{C T} \mid \psi_{f i n}\right\rangle\right)+ \\
& \frac{\partial c_{i n i}^{(2)}\left(c_{i n i}^{(1)}+c_{f i n}^{(1)}\left\langle\psi_{f i n} \mid \psi_{i n i}\right\rangle+c_{C T}^{(1)}\left\langle\psi_{C T} \mid \psi_{i n i}\right\rangle\right)+}{\partial \boldsymbol{R}} \\
& \frac{\partial c_{\mathrm{CT}}^{(2)}}{\partial \boldsymbol{R}}\left(c_{i n i}^{(1)}\left\langle\psi_{i n i} \mid \psi_{C T}\right\rangle+c_{f i n}^{(1)}\left\langle\psi_{f i n} \mid \psi_{C T}\right\rangle+c_{C T}^{(1)}\right)
\end{aligned}
$$

As we mentioned before, the coefficients $c_{i n i}^{(1)}, c_{f i n}^{(2)}$, and $c_{C T}^{(3)}$ are always near the unity, and their gradient is very small. The mixture terms $c_{i n i}^{(2)}$ and $c_{f i n}^{(1)}$ are close to zero. From the diabatic picture, we also know that $\left\langle\psi_{\text {fin }} \mid \psi_{i n i}\right\rangle \rightarrow 0$ and $\left\langle\psi_{C T} \mid \psi_{i n i}\right\rangle=1$. Therefore, the first term in the sum in eq (14) is negligible, and 
we can simplify the second part with the following equation $\left(c_{i n i}^{(1)}+c_{f i n}^{(1)}\left\langle\psi_{f i n} \mid \psi_{i n i}\right\rangle+c_{C T}^{(1)}\left\langle\psi_{C T} \mid \psi_{i n i}\right\rangle\right) \approx 1$. For the third term, because $c_{f i n}^{(1)}=c_{C T}^{(1)}$ and $\left\langle\psi_{f i n} \mid \psi_{C T}\right\rangle=1$, the term $c_{f i n}^{(1)}\left\langle\psi_{f i n} \mid \psi_{C T}\right\rangle$ is negligible compared to $c_{C T}^{(1)}$. The term $\left\langle\psi_{i n i} \mid \psi_{C T}\right\rangle$ and the coefficient term $c_{C T}^{(1)}$ are correlated by the diabatic coupling term $\Delta_{i C T}$

$$
\left.\begin{array}{c}
c_{C T}^{(1)}=\frac{\Delta_{i C T}}{E_{C T}} \\
\Delta_{i C T}=C\left\langle\psi_{i n i} \mid \psi_{C T}\right\rangle
\end{array}\right\} \rightarrow\left\langle\psi_{i n i} \mid \psi_{C T}\right\rangle=\frac{E_{C T}}{C} c_{C T}^{(1)} .
$$

As mentioned before, $C$ is a constant ranging from $3.5 \mathrm{eV}$ to $18 \mathrm{eV}$ depending on the reference. It should be related to the average of the two orbital energies. ${ }^{44}$ Therefore, a value above $10 \mathrm{eV}$ seems to be reasonable, especially when one of the orbital comes from an oxygen or nitrogen atom. Then, for a typical $E_{C T}$ value, this would make $\left\langle\psi_{\text {ini }} \mid \psi_{C T}\right\rangle$ negligible compared to $c_{C T}^{(1)}$. Although it is not always guaranteed that this term can be neglected, we have assumed that it is null to simplify the picture, which is proved to work well during our calculations later.

After all these approximations, the NAC in eq (14) is simplified to a much concise expression

$$
\mathbf{F}_{12} \approx \frac{\partial c_{i n i}^{(2)}}{\partial \boldsymbol{R}} c_{i n i}^{(1)}+\frac{\partial c_{\mathrm{CT}}^{(2)}}{\partial \boldsymbol{R}} c_{C T}^{(1)}
$$

This result tells that only four quantities determine the NAC strength: the coefficients of $\psi_{i n i}$ and $\psi_{C T}$ in the adiabatic state 1 , and the gradient of the coefficient of $\psi_{i n i}$ and $\psi_{C T}$ in the adiabatic state 2 . (We yet recall that $c_{i n i}^{(1)}$ is close to the unity.) Quantum chemical calculation at single point can provide all the coefficient, and just few points more is enough to generate the gradient.

Eq (16) is derived for an ideal three-state model, while in the quantum chemical calculation of real molecules, instead of the coefficient of diabatic states, we may have configuration interaction (CI) coefficients of configuration state functions (CSF), ${ }^{36}$ and more than one CT state is involved. Nevertheless, for the SEIC problems, it is easy to assign the orbitals in the active space to molecule A or B and to 
determine the CSFs that hold the CT character. For the SEIC reaction in the weak-coupling regime, usually the MOs in the active space change very little and their order does not change, if the intermolecular distance does not become very short. Therefore, the character of the CSF is steady and accessible. As a result, we can take a set of CSFs as the approximation of initial, final, and CT states, and generalize eq (16) in the following practical way

$$
\mathbf{F}_{12}^{p \text { Coef }} \equiv \sum_{i \in i n i \in C S F} \frac{\partial c_{i}^{(2)}}{\partial \boldsymbol{R}} c_{i}^{(1)}+\sum_{i \in C T \in C S F} \frac{\partial c_{i}^{(2)}}{\partial \boldsymbol{R}} c_{i}^{(1)}
$$

$\mathbf{F}_{12}^{p \text { Coef }}$ is one of the proxies for NAC that we test later ( $p$ Coef stands for "proxy based on CI cefficients"). It provides us with a way to calculate the NAC from simple quantum chemical calculations. However, the need of gradient of the coefficients is somewhat inconvenient. Can we derive an expression without the gradient? The answer is yes if we take advantage of the expression of the coefficient in eq (12):

$$
\begin{aligned}
\mathbf{F}_{12} \approx & \frac{\partial c_{i n i}^{(2)}}{\partial \boldsymbol{R}} c_{i n i}^{(1)}+\frac{\partial c_{C T}^{(2)}}{\partial \boldsymbol{R}} c_{C T}^{(1)} \\
= & \frac{\partial\left(\frac{-\Delta_{i C T} \Delta_{f C T}}{E_{f i n}\left(E_{C T}-E_{f i n}\right)}\right)}{\partial \boldsymbol{R}} c_{i n i}^{(1)}+\frac{\partial\left(\frac{-\Delta_{f C T}}{E_{C T}-E_{f i n}}\right)}{\partial \boldsymbol{R}} c_{C T}^{(1)} \\
= & \frac{-\frac{\partial \Delta_{i C T}}{\partial \boldsymbol{R}} \Delta_{T}}{E_{f i n}\left(E_{C T}-E_{f i n}\right)} c_{i n i}^{(1)}+\frac{-\Delta_{i C T} \frac{\partial \Delta_{f C T}}{\partial \boldsymbol{R}}}{E_{f i n}\left(E_{C T}-E_{f i n}\right)} c_{i n i}^{(1)}+\frac{-\Delta_{i C T} \Delta_{f C T} \frac{\partial E_{C T}}{\partial \boldsymbol{R}}}{E_{f i n}\left(E_{C T}-E_{f i n}\right)^{2}} c_{i n i}^{(1)} \\
& +\frac{-\frac{\partial \Delta_{f C T}}{\partial \boldsymbol{R}}}{E_{C T}-E_{f i n}} c_{C T}^{(1)}+\frac{-\Delta_{f C T} \frac{\partial E_{C T}}{\partial \boldsymbol{R}}}{\left(E_{C T}-E_{f i n}\right)^{2}} c_{C T}^{(1)}
\end{aligned}
$$


The third and fifth term containing the gradient of CT state energy, which is proportional to the distance, will be negligible compared to other terms. For intermolecular CT coupling, a previous work has concluded that it decreases exponentially along intermolecular distance $R^{49}$ Then, we can take $\frac{\partial \Delta_{x}}{\partial \boldsymbol{R}} \approx \frac{d \Delta_{x}}{d R} \approx A_{x} \Delta_{x}$, where $A_{x}$ is a constant to simplify eq (18) as follows

$$
\begin{aligned}
F_{12} & \approx \frac{-A_{i n i} \Delta_{i C T} \Delta_{f C T}}{E_{f i n}\left(E_{C T}-E_{f i n}\right)} c_{i n i}^{(1)}+\frac{-A_{f i n} \Delta_{f C T} \Delta_{i C T}}{E_{f i n}\left(E_{C T}-E_{f i n}\right)} c_{i n i}^{(1)}-\frac{A_{f i n} \Delta_{f C T}}{E_{C T}-E_{f i n}} c_{C T}^{(1)} \\
& =\left(A_{i n i}+A_{f i n}\right)\left(-\frac{E_{C T}}{E_{f i n}}\right)\left(-\frac{\Delta_{i C T}}{E_{C T}}\right)\left(-\frac{\Delta_{f C T}}{E_{C T}-E_{f i n}}\right) c_{i n i}^{(1)}+A_{f i n}\left(-\frac{\Delta_{f C T}}{E_{C T}-E_{f i n}}\right) c_{C T}^{(1)} \\
& =\left[-\frac{E_{C T}}{E_{f i n}}\left(A_{i n i}+A_{f i n}\right)+A_{f i n}\right] c_{C T}^{(1)} c_{C T}^{(2)} \\
& =\left[-\frac{E_{C T}}{E_{f i n}} A_{i n i}-\frac{E_{C T}-E_{f i n}}{E_{f i n}} A_{f i n}\right] c_{C T}^{(1)} c_{C T}^{(2)} \\
& =\gamma_{C T} c_{C T}^{(1)} c_{C T}^{(2)}
\end{aligned}
$$

where $\gamma_{C T} \equiv E_{f i n}^{-1}\left[-E_{C T} A_{i n i}-\left(E_{C T}-E_{f i n}\right) A_{f i n}\right]$. Note that in eq (19), NAC is a scalar quantity projected along the intermolecular distance. Note also that if we calculate the $F_{21}$ in the same way, we just obtain the same result with opposite sign, $F_{12}=-F_{21}$, which means that our derivation preserves an important property of the NAC.

With eq (19), we find a correlation between the NAC strength and the coefficients of CT states. Conveniently, no coefficient of initial and final states appears. This result bears some similar structure as that in eqs. (7) and (9), where the diabatic coupling is correlated to the orbital overlap between initial/final state and the CT states. The similarity should come from the same superexchange mechanism through CT states, and implies similar performances when the superexchange mechanism is dominant. The term $\gamma_{C T}$ 
is a combination of state energy, $A_{i n i}$, and $A_{f i n}$. Therefore, it is determined by the molecular complex and the intermolecular direction. For a fixed intermolecular direction, we can take $\gamma_{C T}$ as a constant.

In practical terms, to work with CI coefficients of CSFs from a quantum chemical calculation, we should generalize eq (19) to consider all CSF coefficients holding CT character:

$$
F_{12}^{p \operatorname{Coef,2}}=\gamma_{C S F} \kappa_{12}^{C T}
$$

with the definition

$$
\kappa_{12}^{C T} \equiv \sum_{i \in C T \in C S F} c_{i}^{(1)} c_{i}^{(2)}
$$

$\gamma_{C S F}$ groups all other terms dependent on energies and energy derivatives and will be assumed to be a constant for a single incidence direction throughout this paper. For this reason, we will exclusively discuss $\kappa_{I J}^{C T}$, which just requires the coefficient of few CSFs in the adiabatic states $I$ and $J$, which are provided by even just one single point calculation. If eq (21) works well, we can use it to describe the NAC strength for different intermolecular distance within a determined direction.

To summarize, after the derivations based on the adiabatic wave functions of SEIC reaction, we proposed two proxies to express the NAC just adopting coefficients of the wave functions directly from quantum chemical calculations. The first NAC proxy, $\mathbf{F}_{I J}^{p \text { Coef }}$, is given by eq (17) and should deliver the absolute NAC values, based on CI coefficients and CI coefficient gradients. The second NAC proxy, $\kappa_{I J}^{C T}$, is given by eq (21) and should give relative NAC values, based on CI coefficients of CT configurations. As far as we know, these two proxies for NAC are proposed by the first time, and we will test their performance in the next section by comparing them to the first-principle calculated NAC. 


\section{RESULTS AND DISCUSSION}

\subsection{The TF reaction in singlet oxygen photosensitization}

In this work, we take the singlet oxygen photosensitization by thiothymine, an example of triplet fusion reaction (see reaction (3)), as a prototype to study several features in SEIC. The choice of thiothymine as PS holds a couple of advantages: first, the PS- $\mathrm{O}_{2}$ complex is relatively small, so that different quantum chemical levels, including multireference methods, are affordable. Second, $\mathrm{O}_{2}$ has two possible final states, ${ }^{1} \Sigma_{\mathrm{g}}$ and ${ }^{1} \Delta_{\mathrm{g}}$, which delivers valuable additional information on the electronic interactions. This reaction is still far from being satisfactorily treated by theoretical computational chemistry, mainly due to the intrinsic open-shell nature, while new investigations with direct quantum chemical calculation are always welcome..$^{50,51} \mathrm{We}$ explore the developments and conclusions from our previous research on this reaction, ${ }^{11,12}$ to help us focus on the present goal, to test and validate the coupling proxies proposed in the previous sections. Nevertheless, despite the focus on reaction (3), we anticipate that the current findings contribute to a general understanding of SEIC, including SF.

Reaction (3) can generate either ${ }^{1} \Delta_{\mathrm{g}}$ or ${ }^{1} \Sigma_{\mathrm{g}}$ singlet oxygen if the triplet energy $\mathrm{E}_{\mathrm{T}}$ of PS exceeds $\sim 1.6$ $\mathrm{eV}$ (the excitation energy for ${ }^{1} \Sigma_{\mathrm{g}}$ oxygen). For this TF reaction, the initial state is ${ }^{3} \mathrm{PS}-{ }^{3} \mathrm{O}_{2}$ (TT), the final state is ${ }^{1} \mathrm{PS}-{ }^{1} \mathrm{O}_{2}$ (SS), and the CT state to enable the CT-mediated mechanism is either ${ }^{+} \mathrm{PS}^{-} \mathrm{O}_{2}$ or ${ }^{-} \mathrm{PS}^{+} \mathrm{O}_{2}$. In a conventional four-electron view, these states can be described with four frontier molecular orbitals (detailed shown in Section 3.3): the $\pi_{p}$ and $\pi_{p}^{*}$ (the two singly occupied orbital of an organic PS in the $T_{1}$ state) and the $\pi_{x}^{*}$ and $\pi_{y}^{*}$ (the two singly occupied degenerated orbitals of $\mathrm{O}_{2}$ in the ground state).

Although $\mathrm{O}_{2}\left({ }^{1} \Sigma_{\mathrm{g}}\right)$ is quickly deactivated into $\mathrm{O}_{2}\left({ }^{1} \Delta_{\mathrm{g}}\right)$ within solvents, ${ }^{52,53}$ advanced spectroscopic techniques have been developed to distinguish between the two states ${ }^{54,55}$ and provide experimental data for ${ }^{1} \Sigma_{\mathrm{g}} /{ }^{1} \Delta_{\mathrm{g}}$ ratio during singlet oxygen generation. From the computational standpoint, using the DtC model, ${ }^{11}$ we showed that the ${ }^{1} \Sigma_{\mathrm{g}} /{ }^{1} \Delta_{\mathrm{g}}$ rate ratio might range from near to zero up to about 20 , depending on the activation energies $\Delta E^{\ddagger}$ and diabatic coupling strength from different intermolecular incidence 
direction, orientation, and distance between $\mathrm{O}_{2}$ and PS. ${ }^{12} \mathrm{We}$ found that in some directions the ${ }^{1} \Sigma_{\mathrm{g}}$ coupling was always near to zero, while the ${ }^{1} \Delta_{\mathrm{g}}$ coupling is normally large, leading to the relatively small ${ }^{1} \Sigma_{\mathrm{g}}$ $/{ }^{1} \Delta_{\mathrm{g}}$ rate ratio (as plotted in Figure 2 with blue circles).

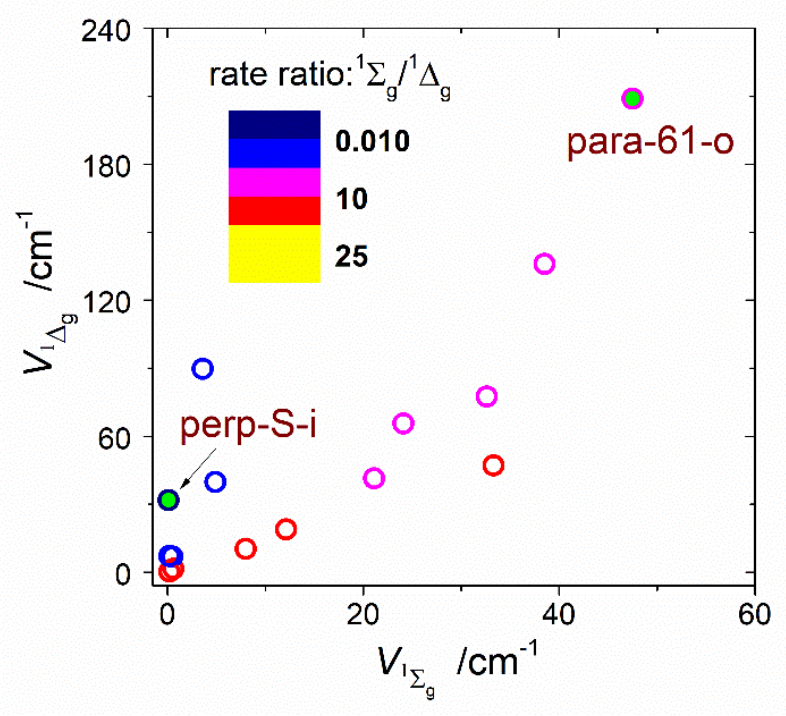

Figure 2. Calculated diabatic couplings for ${ }^{1} \Delta_{\mathrm{g}}$ and ${ }^{1} \Sigma_{\mathrm{g}}$ oxygen generation at the maximum rate distance obtained for 15 incidence directions. The color code indicates the ${ }^{1} \Sigma_{\mathrm{g}} /{ }^{1} \Delta_{\mathrm{g}}$ rate ratio. Results from ref. ${ }^{12}$

Considering the fact that the ${ }^{1} \Delta_{\mathrm{g}}$ and ${ }^{1} \Sigma_{\mathrm{g}}$ oxygens are constructed from the same orbitals with a different spin, ${ }^{51,56}$ the phenomenon of near zero ${ }^{1} \Sigma_{\mathrm{g}}$ coupling but much larger ${ }^{1} \Delta_{\mathrm{g}}$ coupling is intriguing. Nevertheless, in that previous computational study, we did not directly discuss the underlying mechanism responsible for it, while in this work it will be revealed in detail, thanks to the theoretical tools proposed in Section 2. In what follows, we picked out two points among the fifteen incidence directions_- "perp-S-i" (for which the ${ }^{1} \Sigma_{\mathrm{g}}$ coupling is little) and "para-61-o" (for which both couplings are large)—and restrict the analysis to them. These two directions are indicated in Figure 2 by the green filled circles (their geometries are shown in the SI). 


\subsection{Analysis in the adiabatic picture}

In this section, we test the performance of the NAC proxies given by eqs (17) and (21) in comparison to the full NAC directly computed at the CASSCF level for the $\mathrm{PS}_{-} \mathrm{O}_{2}$ complex. During the whole calculation, the orbitals from PS and $\mathrm{O}_{2}$ do not mix much, so it is easy to designate the four MOs in the active space to $\pi_{p}^{*}, \pi_{p}, \pi_{x}^{*}$ and $\pi_{y}^{*}$ respectively. These MOs for the two directions are plotted in Figure 3.

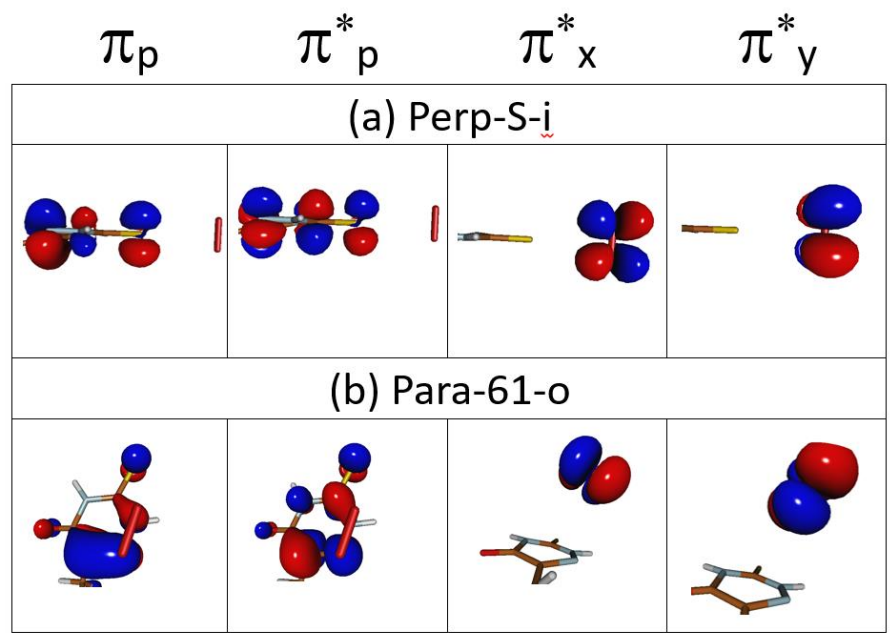

Figure 3. The four frontier molecular orbitals from the CASSCF calculation of PS-O ${ }_{2}$ along (a) perp-S-i and (b) para-61-o directions.

With these MOs, we determined the four CSFs that carry the CT characters according to the electron occupation of the CSFs. Their CI coefficients in different adiabatic states were obtained after the CASSCF calculation. The initial state is the adiabatic state $\mathrm{S}_{4}$, and the final state may be any of the adiabatic states $S_{1}$ to $S_{3}$. The final states $S_{1}$ and $S_{2}$ correspond to the generation of ${ }^{1} \Delta_{g}$ oxygen, while the final state $S_{3}$ corresponds to the generation of ${ }^{1} \Sigma_{\mathrm{g}}$ oxygen. We took eqs (17) and (21) to calculate $\mathbf{F}_{I J}^{p C o e f}$ and $\kappa_{I J}^{C T}$, and investigated their performance as the NAC proxy quantitatively along the intermolecular distance.

According to eq (17), the coefficients of the CT and TT CSFs in the initial state as well as their gradient are needed to calculate $\mathbf{F}_{I J}^{p C o e f}$. Ten CSFs with the largest weights in the initial state $\mathrm{S}_{4}$ at the first point (with longest intermolecular distance) were taken as the TT CSFs. We are interested in the projection 
$F_{I J}^{p C o e f}$ of the NAC vector on the intermolecular distance. Thus, the gradient is simply the derivative along the intermolecular coordinate, and it was calculated by finite difference along the linearly interpolated pathway:

$$
\frac{d c_{i}^{I}\left(R_{n}\right)}{d R} \approx \frac{c_{i}^{I}\left(R_{n+1}\right)-c_{i}^{I}\left(R_{n-1}\right)}{2 \Delta R}
$$

where $\Delta R$ is step length used for the calculation of this direction. Following eq (21), the calculation of $\kappa_{I J}^{C T}$ is simpler and only requires the CI coefficients of the four CT CSFs. The calculated $F_{I J}^{p C o e f}$ and $\kappa_{I J}^{C T}$ are compared with the first-principles NAC $F_{I J}^{D C}$ (DC stands for "directly computed"), which is the projection of the NAC vector on the intermolecular direction $\mathbf{R}$.

We start the analysis with the directions para-61-o, whose results are plotted in Figure 4. The excellent performance of $F_{I J}^{p C o e f}$ is presented in Figure 4(a). The $F_{I J}^{p C o e f}$ of three reaction paths are very close to $F_{I J}^{D C}$, especially when the intermolecular distance is around or larger than the distance of maximum rate point, which is always larger than $2.9 \AA .{ }^{12}$ When the molecules get closer, the deviation might increase, although the approximation still works well for some of the couplings. The deviation may come from taking the CSFs as the approximation of diabatic states and supposing they are constant. However, the small distance will increase the activation energy a lot and make it unimportant for the final reaction rate. So, $F_{I J}^{p C o e f}$ works very well as a proxy for NAC. 

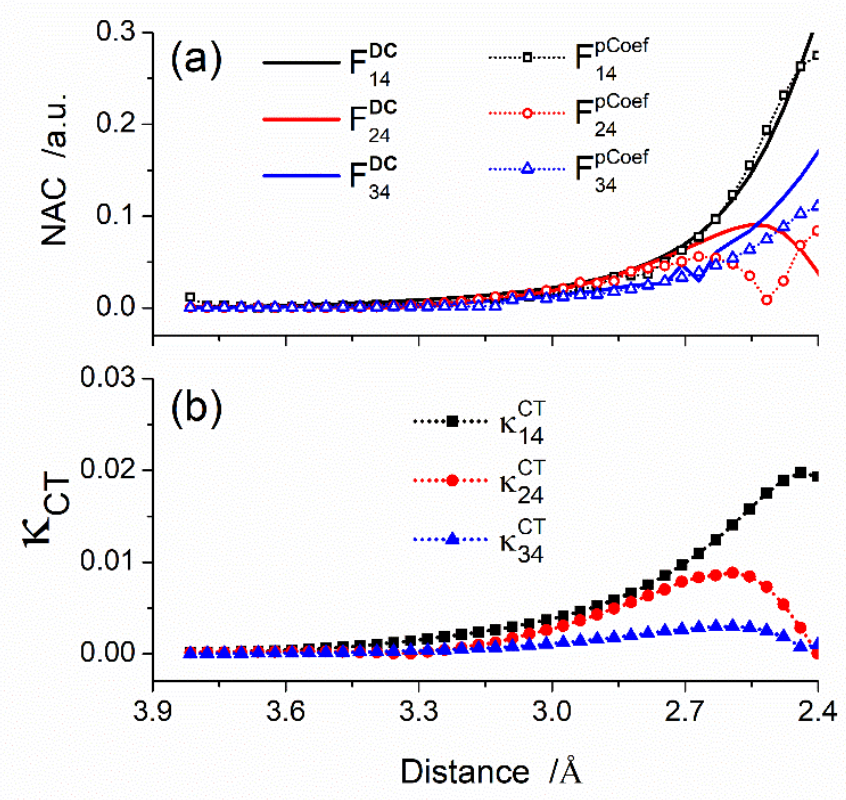

Figure 4. (a) First-principle NAC $F_{S S-T T}^{D C}$, the proxy $F_{I J}^{p \text { Coef }}$ from eq (17), and (b) the proxy $\kappa_{I J}^{C T}$ from eq (21) along the para-61-o direction. The distance of maximum rate is $2.90 \AA .^{12}$

The results in Figure 4(b) also confirm the correlation between $\kappa_{I J}^{C T}$ and $F_{I J}^{D C}$, with all the three lines present the same trends as those of $F_{I J}^{D C}$, if the intermolecular distance is not too small. So $\kappa_{I J}^{C T}$ can tell the trend of NAC strength along the intermolecular distance and become a good proxy for NAC. We note here that $\kappa_{I J}^{C T}$ does not have the same unit as NAC, and strengths from this proxy are only comparable when the molecules and related adiabatic states are the same.

Now, we analyze the perp-S-i direction where the ${ }^{1} \Sigma_{\mathrm{g}}$ coupling is tiny. The results are presented in Figure 5. Again, both $F_{I J}^{p C o e f}$ and $\kappa_{I J}^{C T}$ have excellent performances as proxies for NAC. They generated similar shapes along the intermolecular distance, and $F_{I J}^{p \text { Coef }}$ gives nearly the same value as the directly calculated NAC, if the intermolecular distance is not too small. 

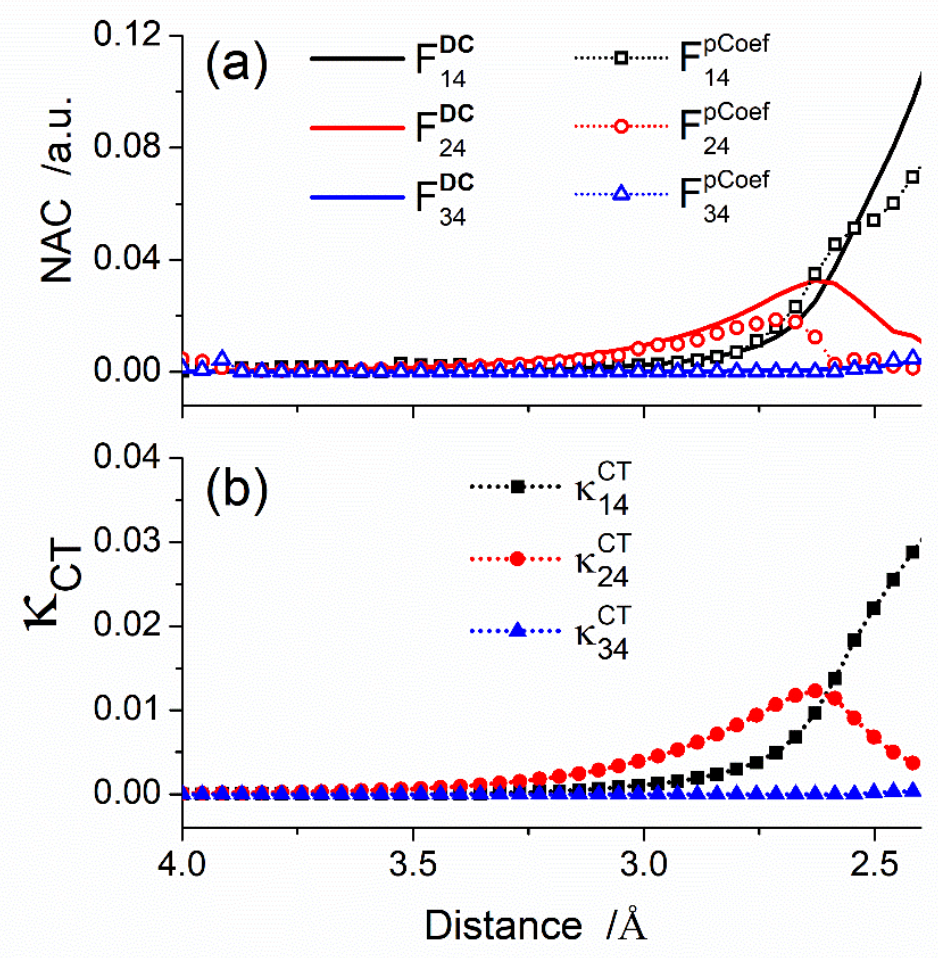

Figure 5. (a) First-principle NAC $F_{S S-T T}^{D C}$, the proxy $F_{I J}^{p C o e f}$ from eq (17), and (b) the proxy $\kappa_{I J}^{C T}$ from eq (21) along the perp-S-i direction. The distance of the maximum rate is $3.14 \AA .^{12}$

The use of $F_{I J}^{p \text { Coef }}$ and $\kappa_{I J}^{C T}$ as NAC proxies goes beyond to have an inexpensive estimate of the NAC. Unlike the full NAC, which is difficult to analyze directly, these two proxies are determined by simple equations, being much easier to interpret, especially $\kappa_{I J}^{C T}$. For instance, the proxies tell us why perp-S-i directions have tiny ${ }^{1} \Sigma_{\mathrm{g}}$ couplings. As shown in Figure $5, \kappa_{34}^{C T}$ as well as $F_{34}^{D C}$ are always near zero along the intermolecular distance. With its definition in eq (21), we know that $\kappa_{34}^{C T}$ is determined by the coefficient of CT CSFs in $\mathrm{S}_{3}$ and $\mathrm{S}_{4}$. We already have all of these coefficients in hand from the CASSCF calculation, so it is forthright to determine why $\kappa_{34}^{C T}=\sum_{i \in C T \in C S F} c_{i}^{(3)} c_{i}^{(4)}$ is always near to zero: it occurs due to the complementary distribution of the four involved CT CSFs (configurations shown in next subsection) on $\mathrm{S}_{3}$ and $\mathrm{S}_{4}$. 
For some CT configurations, $\left|c_{i}^{(3)}\right|>0$ but $c_{i}^{(4)} \approx 0$, while for others it is just the opposite, $\left|c_{i}^{(4)}\right|>0$ but $c_{i}^{(3)} \approx 0$. As a result, each term in the sum $\sum_{i \in C R \in C S F} c_{i}^{(3)} c_{i}^{(4)}$ is near zero. In other words, some CT CSFs are involved in $\mathrm{S}_{4}$, but they do not appear in $\mathrm{S}_{3}$, while for others, it is just the opposite. Therefore, although the CT configurations are not absent in both adiabatic states, there are no CT configurations that can connect the two states successfully.

Why is the nonadiabatic coupling zero only for $S_{3}$, not for $S_{1}$ or $S_{2}$ state? As shown in eq (12), the coefficients of the CT CSFs in these two adiabatic states are determined by the coupling strength terms $\Delta_{i C T}$ and $\Delta_{f C T}$, which are the electronic couplings between the intermediate CT state and the initial TT and the final SS states, respectively. Then, the distribution of the four CT CSFs on $\mathrm{S}_{3}$ and $\mathrm{S}_{4}$ is transformed to the diabatic coupling problem: no CT configuration has a large coupling with both the initial TT and final ${ }^{1} \sum_{\mathrm{g}} \mathrm{SS}$ state. As we discussed in Subsection 2.1, these coupling strengths can be evaluated by the orbital overlaps, and we already build a correlation between the total SEIC diabatic coupling and the product of two orbital overlap term in eq (8). Therefore, it is more convenient to study this problem in the diabatic picture, as we do in next subsection.

\subsection{Analysis in the diabatic picture}

In the diabatic picture, we need to consider the initial TT state and final SS diabatic states and the CT mediated interaction paths between them. To keep the analysis in a clear and practical way, we investigate the interaction path based on the configurations directly constructed with frontier orbitals. For the initial ${ }^{1} \mathrm{TT}$ state from the product of triplet state of PS $\left(\mathrm{T}_{1}\right)$ and triplet state of $\mathrm{O}_{2}\left({ }^{3} \Sigma_{g}\right)$, the wave function of the complex is a linear combination of configurations whose the spin projection of PS and $\mathrm{O}_{2}$ sum to zero: ${ }^{1}$

$$
\psi_{P S-O_{2}}\left(\mathrm{~T}_{1}-{ }^{3} \Sigma_{g}\right)=\frac{1}{\sqrt{3}}\left\{\mathrm{~T}_{+} \mathrm{T}_{-}+\mathrm{T}_{-} \mathrm{T}_{+}-\mathrm{T}_{0} \mathrm{~T}_{0}\right\}
$$


In the $(4,4)$ space, $\mathrm{T}_{+} \mathrm{T}_{-}$and $\mathrm{T}_{-} \mathrm{T}_{+}$configurations are represented by single determinants (Figure 6 ), while $\mathrm{T}_{0} \mathrm{~T}_{0}$ is written in terms of four determinants.

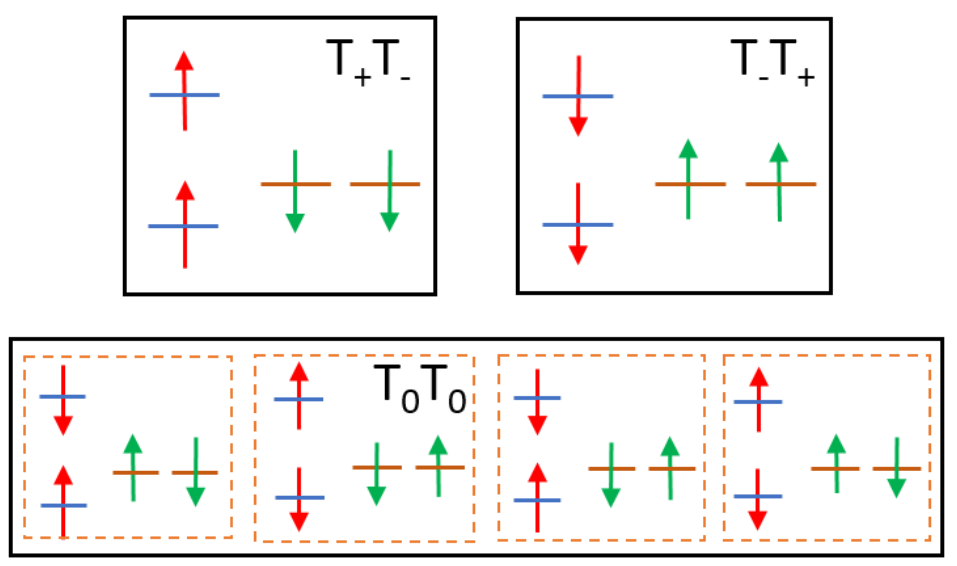

Figure 6. The electronic configurations for the ${ }^{1} \mathrm{TT}$ state of $\mathrm{PS}-\mathrm{O}_{2}$ with the four frontier orbitals.

For the final ${ }^{1} \mathrm{SS}$ states, the wave function of the complex is written in terms of the product of the closeshell ground state of PS $\left(\mathrm{S}_{0}\right)$ and the singlet state of $\mathrm{O}_{2}\left({ }^{1} \Sigma_{\mathrm{g}}\right.$ or $\left.{ }^{1} \Delta_{\mathrm{g}}\right)$. In the $(2,2)$ space of $\mathrm{O}_{2}$, these wafve functions are: $:^{51,57}$

$$
\begin{aligned}
& \psi_{O_{2}}\left(\mathrm{~S}_{0}-{ }^{1} \Delta_{g 0}\right)=\left|\mathrm{S}_{0}\right\rangle \otimes \frac{1}{\sqrt{2}}\left\{\left|\pi_{x}^{*}(1) \bar{\pi}_{\mathrm{y}}^{*}(2)\right\rangle-\left|\pi_{y}^{*}(1) \bar{\pi}_{x}^{*}(2)\right\rangle\right\}=\mathrm{SS} 1-\mathrm{SS} 3 \\
& \psi_{O_{2}}\left(\mathrm{~S}_{0}-{ }^{1} \Delta_{g 1}\right)=\left|\mathrm{S}_{0}\right\rangle \otimes \frac{1}{\sqrt{2}}\left\{\left|\pi_{x}^{*}(1) \bar{\pi}_{x}^{*}(2)\right\rangle-\left|\pi_{y}^{*}(1) \bar{\pi}_{y}^{*}(2)\right\rangle\right\}=\mathrm{SS} 2-\mathrm{SS} 4 \\
& \psi_{O_{2}}\left(\mathrm{~S}_{0}-{ }^{1} \Sigma_{g}\right)=\left|\mathrm{S}_{0}\right\rangle \otimes \frac{1}{\sqrt{2}}\left\{\left|\pi_{x}^{*}(1) \bar{\pi}_{x}^{*}(2)\right\rangle+\left|\pi_{y}^{*}(1) \bar{\pi}_{y}^{*}(2)\right\rangle\right\}=\mathrm{SS} 2+\mathrm{SS} 4
\end{aligned}
$$

where SS1 for instance is $\left|S_{0}\right\rangle \otimes \frac{1}{\sqrt{2}}\left|\pi_{x}^{*}(1) \bar{\pi}_{\mathrm{y}}^{*}(2)\right\rangle$ and SS1 to SS4 are illustrated at the right side of Figure 7.

For the CT-mediated mechanism, we also need to define the CT configurations (CT1 to CT4), which are illustrated in the middle of Figure 7. In a way analogous to Figure 1, we generate interaction paths between all TT and all SS configurations through CT configurations. Taking the $\mathrm{T}_{+} \mathrm{T}_{-}$configuration, for example, all interaction paths connecting it to SS1 to SS4 are shown in Figure 7. The diabatic couplings 
between configurations can now be written. For instance, based on eq (9), the coupling term of $\mathrm{T}_{+} \mathrm{T}_{-}$with SS1 through CT1 (the first pathway in Figure 7) is

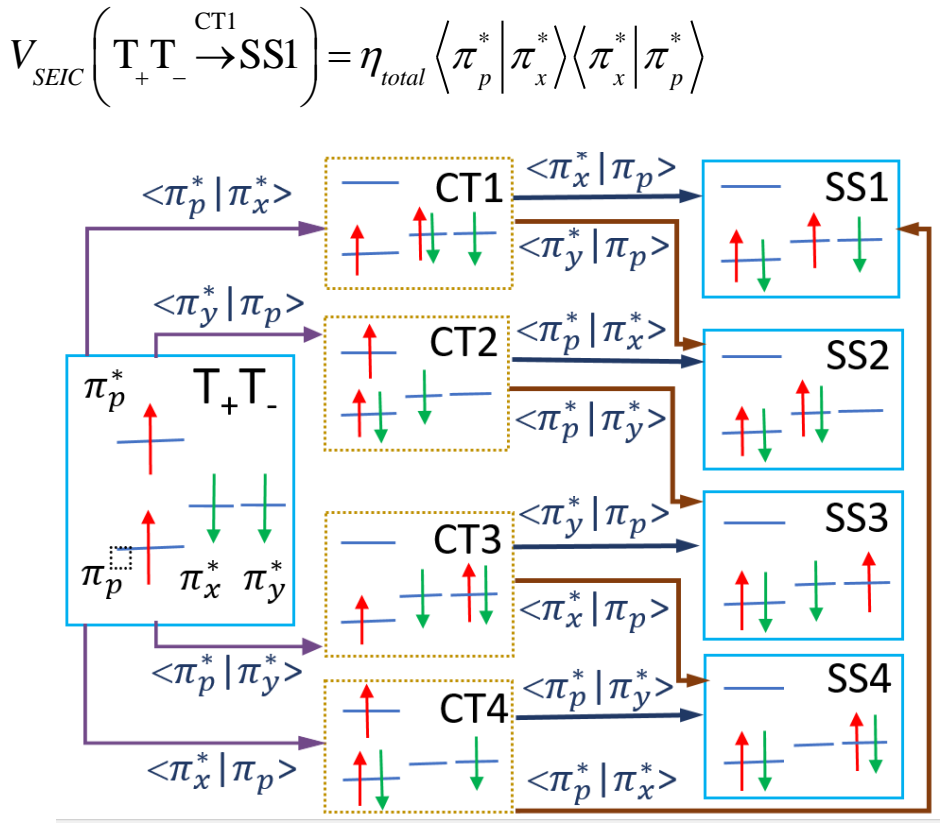

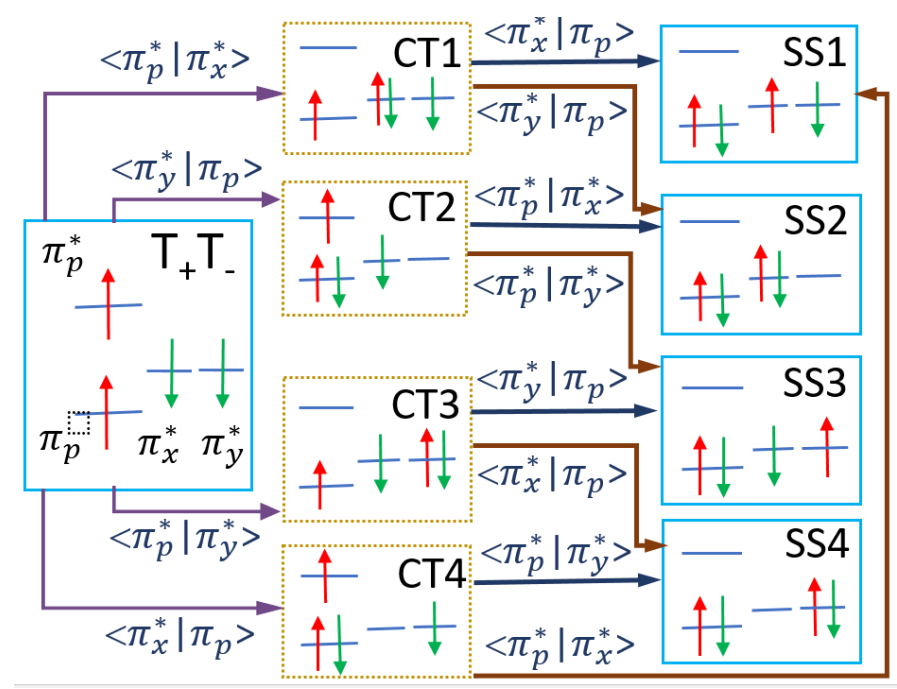

Figure 7. Interaction paths based on CT-mediated mechanism for triplet fusion of $\mathrm{PS}-\mathrm{O}_{2}$ system from the

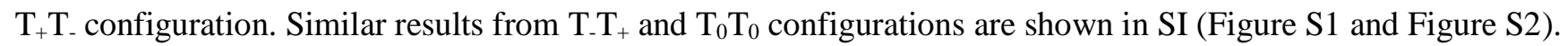

As we mentioned before, the zero ${ }^{1} \Sigma_{\mathrm{g}}$ coupling along perp-S-i direction is intriguing. As shown in Figure $8(\mathrm{a})$, the ${ }^{1} \sum_{\mathrm{g}}$ coupling along this direction is always near to zero, while the other two diabatic coupling strengths increase when the intermolecular distance decreases. We can now use the diabatic coupling given in eq (9) to explain the reason underlying this effect.

From eq (24), we know that the only the SS2 and SS4 configurations are needed for ${ }^{1} \Sigma_{\mathrm{g}}$ singlet oxygen generation. As shown in Figure 7, the diabatic coupling from $\mathrm{T}_{+} \mathrm{T}_{-}$to $\mathrm{SS} 2$ through either $\mathrm{CT} 1$ or $\mathrm{CT} 2$ is proportional to the MO overlap term $\left\langle\pi_{p}^{*} \mid \pi_{x}^{*}\right\rangle\left\langle\pi_{y}^{*} \mid \pi_{p}\right\rangle$. (CT3 and CT4 do not contribute to this coupling.) the diabatic coupling from $\mathrm{T}_{+} \mathrm{T}_{-}$to SS4 through either CT3 or CT4 is proportional to $\left\langle\pi_{p}^{*} \mid \pi_{y}^{*}\right\rangle\left\langle\pi_{x}^{*} \mid \pi_{p}\right\rangle$ (without contributions from CT1 or CT2). If we further consider the diabatic couplings from $\mathrm{T}_{-} \mathrm{T}_{+}$or $\mathrm{T}_{0} \mathrm{~T}_{0}$ 
configurations using the same procedure (Figure S1 and Figure S2 in the SI), we see that only the same overlap terms are essential for the ${ }^{1} \Sigma_{\mathrm{g}}$ singlet oxygen generation.
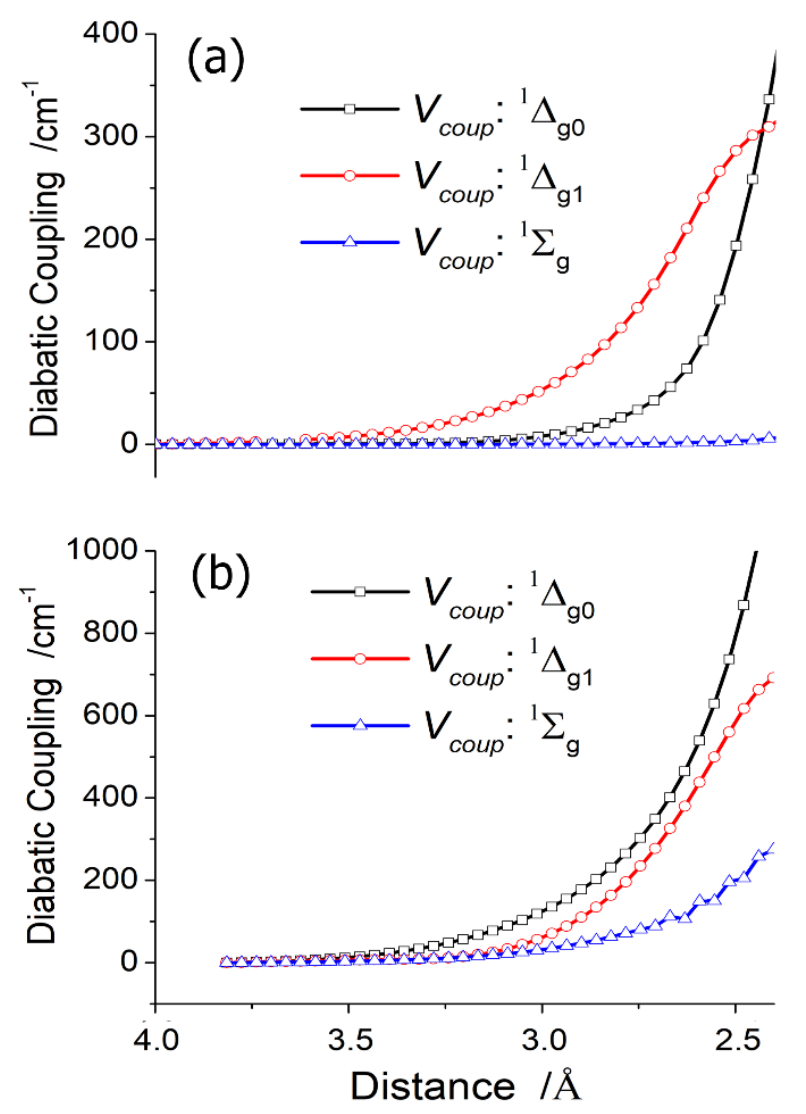

Figure 8. Calculated diabatic couplings for ${ }^{1} \Delta_{\mathrm{g}}$ and ${ }^{1} \sum_{\mathrm{g}}$ oxygen generation along the (a) perp-S-i and (b) para61-o direction.

With the four MOs from the CASSCF calculations plotted in Figure 3(a), we can estimate the magnitude of these overlap terms for the perp-S-i direction. The overlap occurs along the sulfur- $\mathrm{O}_{2}$ direction, and the orbital $\pi_{p}^{*}$ and $\pi_{p}$ around the sulfur atom are perfectly oriented to maximize the overlap with $\pi_{x}^{*}$, but minimize the overlap with $\pi_{y}^{*}$. Thus, both $\left\langle\pi_{y}^{*} \mid \pi_{p}^{*}\right\rangle$ and $\left\langle\pi_{y}^{*} \mid \pi_{p}\right\rangle$ tend to be tiny. With these two terms near to zero, both overlap terms $\left\langle\pi_{p}^{*} \mid \pi_{x}^{*}\right\rangle\left\langle\pi_{y}^{*} \mid \pi_{p}\right\rangle$ and $\left\langle\pi_{p}^{*} \mid \pi_{y}^{*}\right\rangle\left\langle\pi_{x}^{*} \mid \pi_{p}\right\rangle$ become very small, which means the interaction through CT-mediated mechanism is not effective for both SS2 and SS4 configurations, explaining the zero ${ }^{1} \Sigma_{\mathrm{g}}$ coupling along this direction. 
According to the wave function for PS-O $2\left({ }^{1} \mathrm{~S}_{0}-{ }^{1} \Delta_{g}\right)$ state in eq (24), the ${ }^{1} \Delta_{\mathrm{g} 1}$ coupling also depends on the SS2 and SS4 configurations, and it should be near to zero too. On the other hand, the ${ }^{1} \Delta_{\mathrm{g} 0}$ coupling is determined by the overlap terms with the SS1 and SS3 configurations, $\left\langle\pi_{p}^{*} \mid \pi_{x}^{*}\right\rangle\left\langle\pi_{x}^{*} \mid \pi_{p}\right\rangle$ and $\left\langle\pi_{p}^{*} \mid \pi_{y}^{*}\right\rangle\left\langle\pi_{y}^{*} \mid \pi_{p}\right\rangle$. Although $\left\langle\pi_{p}^{*} \mid \pi_{y}^{*}\right\rangle\left\langle\pi_{y}^{*} \mid \pi_{p}\right\rangle$ should be small, $\left\langle\pi_{p}^{*} \mid \pi_{x}^{*}\right\rangle\left\langle\pi_{x}^{*} \mid \pi_{p}\right\rangle$ should have a large overlap value, implying that the ${ }^{1} \Delta_{\mathrm{g} 0}$ coupling should be much larger than that for ${ }^{1} \Sigma_{\mathrm{g}}$.

Nevertheless, the NACs for adiabatic states $S_{1}$ and $S_{2}$ states of perp-S-i are both not null, because both $\mathrm{S}_{1}$ and $\mathrm{S}_{2}$ states are not pure ${ }^{1} \Delta_{\mathrm{g} 1}$ or ${ }^{1} \Delta_{\mathrm{g} 0}$, but a mixture of them. To prove this point, we analyzed the components of these two states. If the coupling for ${ }^{1} \Delta_{\mathrm{g} 1}$ is near zero, the coupling strength for the mixed state should only depend on the ${ }^{1} \Delta_{\mathrm{g} 0}$ contribution. We took the ${ }^{1} \Delta_{\mathrm{g} 0}$ coefficients of the $\mathrm{S}_{1}$ and $\mathrm{S}_{2}$ states and calculated the ratio between them. This coefficient ratio was compared to the ratio of the NAC, as plotted in Figure 9. These two ratios are nearly the same, strongly supporting our interpretation.

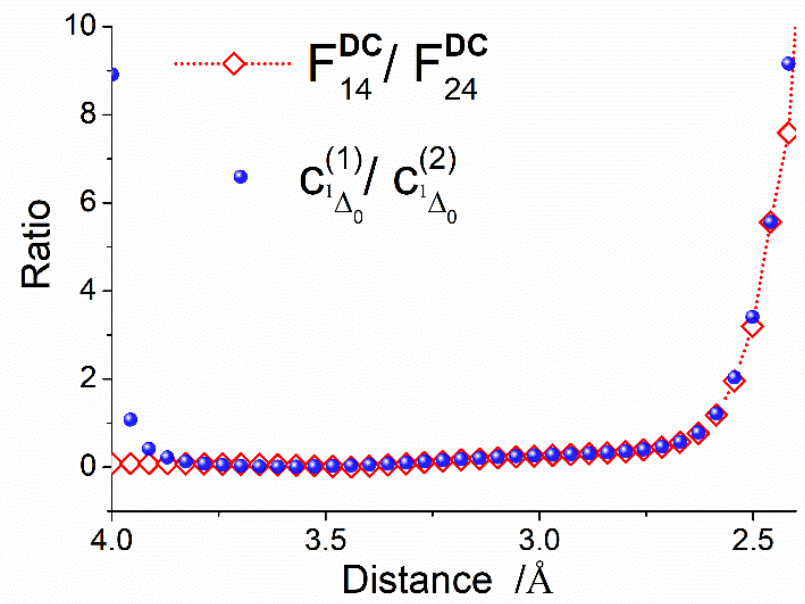

Figure 9. The ratios of NAC and ${ }^{1} \Delta_{\mathrm{g} 0}$ coefficient of adiabatic $\mathrm{S}_{1}$ and $\mathrm{S}_{2}$ states for the perp-S-i direction.

For the para-61-o direction, the orbitals were taken from Figure 3(b). Here, both $\pi_{x}^{*}$ and $\pi_{y}^{*}$ are not perpendicular or parallel to the ring plane but are sloped. Consequently, all overlaps are not zero, and ${ }^{1} \Sigma_{\mathrm{g}}$ coupling is larger than zero, which agrees with the fully calculated coupling as shown in Figure 8(b). 


\section{CONCLUSION}

Spin-exchange internal conversion (SEIC) is a class of reactions including singlet fission (SF) and triplet fusion (TF) as particular cases. Based on the understanding of SEIC reaction, we proposed proxies to evaluate the coupling strength in reliable but simple ways, and tested them on the PS-O $\mathrm{O}_{2}$ singlet oxygen generation problem, an example of the TF reaction. In the diabatic picture, we built a bilinear relationship between the diabatic coupling and a product of two orbital overlap terms, and applied it together with related MOs to interpret the curious behavior of diabatic coupling along some intermolecular directions; in the adiabatic picture, two proxies of the NAC related to the CI coefficients of specific CSFs were proposed, and their results were compared with the directly calculated full NAC. Only the singlet oxygen photosensitization from the $\mathrm{T}_{1}$ state of PS was investigated because of the fast and high quantum-yield relaxation to $T_{1}$ state after photoexcitation of PS. In the case of long-lived higher excited states, a weakcoupling reaction could still be treated with a similar approach, while other reactions may need additional methodological developments.

In the adiabatic picture, the full NAC and the two NAC proxies were computed along the PS-O 2 intermolecular distance. The excellent performance of both proxies on this reaction system is confirmed from the direct comparison with the full NAC. The proxies are derived for a general SEIC reaction, which implies that we can employ them to make inexpensive semiquantitative analysis for SEIC reactions in large molecular ensembles and aid the qualitative analysis of the interstate interaction, under the conditions that the coupling from CT-mediated mechanism is dominant compared to that from the direct mechanism.

In the diabatic picture, we have employed the diabatic coupling proxy based on the orbital overlap term to qualitatively explain why the ${ }^{1} \Sigma_{\mathrm{g}}$ coupling is always zero in some incidence directions of the PS-O ${ }_{2}$ reaction. There are still some challenges, including the exact computation of the CT state energy and 
orbital overlap, to quantitatively calculate the diabatic coupling value, which remains for the future research. However, the evaluation from orbital overlap could be a simple way to predict the coupling strength along the various intermolecular directions, providing practical guideline for related molecular design.

The new proxies in adiabatic and diabatic pictures have their own advantages. For NAC proxies, they are calculated based on quantum chemical calculations and provide robust results comparable to the full NAC strength. On the other hand, the orbital overlaps in the diabatic picture are easier to analyze even without resorting to explicit calculations. In the example studied here, just the shapes of four key MOs were enough to deliver a qualitative analysis. Although the MOs used here are from CASSCF calculations, the analysis should be valid with Kohn-Sham orbitals even from isolated molecules as well. It is straightforward to extend these analyses to other SEIC problems with larger systems, if the dominant frontier MOs for the related excited states during the reaction are already known.

The approaches are derived for general SEIC reactions, although we take one special TF reaction case to prove the validity. Since photosensitization of singlet oxygen generation is an important research topic for experiments and theorists, ${ }^{50}$ the current findings during the verification process are also timely and appealing to researchers in this field.

\section{ASSOCIATED CONTENT}

\section{Supporting Information}

Details on quantum chemical calculation, molecular orbitals in the active spaces. This material is available free of charge via the Internet at http://pubs.acs.org.

\section{AUTHOR INFORMATION}

\section{Corresponding Author}

\section{E-mail: shuming.bai@univ-amu.fr}


E-mail: mario.barbatti@univ-amu.fr; website:www.barbatti.org

\section{Notes}

The authors declare no competing financial interests.

\section{ACKNOWLEDGMENT}

We are grateful to Prof. Hans Lischka and Dr. Felix Plasser for discussions and valuable suggestions. This work was supported by Excellence Initiative of Aix-Marseille University (A*MIDEX) and the project Equip@Meso (ANR-10-EQPX-29-01), both funded by the French Government "Investissements d'Avenir" program. The authors also acknowledge funding from HPC resources from GENCI-CINES (Grant 2017-A0010810012). MB also acknowledges funding from and of the WSPLIT project (ANR-17CE05-0005-01).

\section{REFERENCES}

1. Casanova, D., Theoretical Modeling of Singlet Fission. Chem. Rev. 2018, 118 (15), 7164-7207.

2. Zimmerman, P. M.; Musgrave, C. B.; Head-Gordon, M., A Correlated Electron View of Singlet Fission. Acc. Chem. Res. 2013, 46 (6), 1339-1347.

3. Zimmerman, P. M.; Bell, F.; Casanova, D.; Head-Gordon, M., Mechanism for Singlet Fission in Pentacene and Tetracene: From Single Exciton to Two Triplets. J. Am. Chem. Soc. 2011, 133 (49), 1994419952.

4. Smith, M. B.; Michl, J., Singlet Fission. Chem. Rev. 2010, 110 (11), 6891-6936.

5. Busby, E.; Xia, J. L.; Wu, Q.; Low, J. Z.; Song, R.; Miller, J. R.; Zhu, X. Y.; Campos, L. M.; Sfeir, M. Y., A design strategy for intramolecular singlet fission mediated by charge-transfer states in donor-acceptor organic materials. Nat. Mater. 2015, 14 (4), 426-433.

6. Paci, I.; Johnson, J. C.; Chen, X. D.; Rana, G.; Popovic, D.; David, D. E.; Nozik, A. J.; Ratner, M. A.; Michl, J., Singlet fission for dye-sensitized solar cells: Can a suitable sensitizer be found? J. Am. Chem. Soc. 2006, 128 (51), 16546-16553. 
7. Abdel-Shafi, A. A.; Wilkinson, F., Electronic to vibrational energy conversion and charge transfer contributions during quenching by molecular oxygen of electronically excited triplet states. Phys. Chem. Chem. Phys. 2002, 4 (2), 248-254.

8. Schmidt, R., Quantitative determination of (1)Sigma(+)(g) and (1)Delta(g) singlet oxygen in solvents very different polarity. General energy gap law for rate constants of electronic energy transfer to and from O-2 in the absence of charge-transfer interactions J. Phys. Chem. A 2006, 110 (34), 2622-2628.

9. Kondakov, D. Y.; Pawlik, T. D.; Hatwar, T. K.; Spindler, J. P., Triplet annihilation exceeding spin statistical limit in highly efficient fluorescent organic light-emitting diodes. J. Appl. Phys. 2009, 106 (12), 124510 .

10. Jia, W. Y.; Chen, Q. S.; Chen, L. X.; Yuan, D.; Xiang, J.; Chen, Y. B.; Xiong, Z. H., Molecular Spacing Modulated Conversion of Singlet Fission to Triplet Fusion in Rubrene-Based Organic LightEmitting Diodes at Ambient Temperature. J. Phys. Chem. C 2016, 120 (15), 8380-8386.

11. Bai, S.; Barbatti, M., Divide to Conquer: A Kinetic Model for Singlet Oxygen Photosensitization. J. Chem. Theory Comput. 2017, 13 (11), 5528-5538.

12. Bai, S.; Barbatti, M., Spatial Factors for Triplet Fusion Reaction of Singlet Oxygen

Photosensitization. J. Phys. Chem. Lett. 2017, 8, 5456-5460.

13. Schweitzer, C.; Mehrdad, Z.; Noll, A.; Grabner, E. W.; Schmidt, R., Mechanism of photosensitized generation of singlet oxygen during oxygen quenching of triplet states and the general dependence of the rate constants and efficiencies of O-2((1)Sigma $(+)(g))$, O-2((1)Delta(g)), and O2((3)Sigma(-)(g)) formation on sensitizer triplet state energy and oxidation potential. J. Phys. Chem. A 2003, 107 (13), 2192-2198.

14. Schmidt, R., The Effect of Solvent Polarity on the Balance between Charge Transfer and NonCharge Transfer Pathways in the Sensitization of Singlet Oxygen by $\pi \pi^{*}$ Triplet States. J. Phys. Chem. A 2006, $110(18), 5990-5997$. 
15. Merrifield, R. E., Theory of Magnetic Field Effects on the Mutual Annihilation of Triplet Excitons J. Chem. Phys. 1968, 48 (9), 4318.

16. Jortner, J.; Rice, S. A.; Katz, J. L.; Choi, S., Triplet Excitons in Crystals of Aromatic Molecules. J. Chem. Phys. 1965, 42, 309-323.

17. Yost, S. R.; Lee, J.; Wilson, M. W. B.; Wu, T.; McMahon, D. P.; Parkhurst, R. R.; Thompson, N. J.; Congreve, D. N.; Rao, A.; Johnson, K.; Sfeir, M. Y.; Bawendi, M. G.; Swager, T. M.; Friend, R. H.; Baldo, M. A.; Van Voorhis, T., A transferable model for singlet-fission kinetics. Nat. Chem. 2014, $6(7), 492-497$.

18. Feng, X. T.; Luzanov, A. V.; Krylov, A. I., Fission of Entangled Spins: An Electronic Structure Perspective. J. Phys. Chem. Lett. 2013, 4 (22), 3845-3852.

19. Yang, C. H.; Hsu, C. P., First-Principle Characterization for Singlet Fission Couplings. J. Phys. Chem. Lett. 2015, 6 (10), 1925-1929.

20. Serrano-Perez, J. J.; Olaso-Gonzalez, G.; Merchan, M.; Serrano-Andres, L., Singlet oxygen generation in PUVA therapy studied using electronic structure calculations. Chem. Phys. 2009, 360 (13), 85-96.

21. Greyson, E. C.; Stepp, B. R.; Chen, X. D.; Schwerin, A. F.; Paci, I.; Smith, M. B.; Akdag, A.; Johnson, J. C.; Nozik, A. J.; Michl, J.; Ratner, M. A., Singlet Exciton Fission for Solar Cell Applications Energy Aspects of Interchromophore Coupling. J. Phys. Chem. B 2010, 114 (45), 14223-14232.

22. Smith, M. B.; Michl, J., Recent Advances in Singlet Fission. In Annual Review of Physical Chemistry, Johnson, M. A.; Martinez, T. J., Eds. 2013; Vol. 64, pp 361-386.

23. Beljonne, D.; Yamagata, H.; Bredas, J. L.; Spano, F. C.; Olivier, Y., Charge-Transfer Excitations Steer the Davydov Splitting and Mediate Singlet Exciton Fission in Pentacene. Phys. Rev. Lett. 2013, 110 (22), 226402. 
24. Monahan, N.; Zhu, X. Y., Charge Transfer-Mediated Singlet Fission. In Annual Review of Physical Chemistry, Vol 66, Johnson, M. A.; Martinez, T. J., Eds. 2015; Vol. 66, pp 601-618.

25. Grofe, A.; Qu, Z.; Truhlar, D. G.; Li, H.; Gao, J., Diabatic-At-Construction Method for Diabatic and Adiabatic Ground and Excited States Based on Multistate Density Functional Theory. J. Chem. Theory Comput. 2017, 13 (3), 1176-1187.

26. Wu, Q.; Cheng, C.-L.; Van Voorhis, T., Configuration interaction based on constrained density functional theory: A multireference method. J. Chem. Phys. 2007, 127 (16), 164119.

27. Varga, Z.; Parker, K. A.; Truhlar, D. G., Direct diabatization based on nonadiabatic couplings: the N/D method. Phys. Chem. Chem. Phys. 2018, 20 (41), 26643-26659.

28. Van Voorhis, T.; Kowalczyk, T.; Kaduk, B.; Wang, L. P.; Cheng, C. L.; Wu, Q., The Diabatic Picture of Electron Transfer, Reaction Barriers, and Molecular Dynamics. In Annual Review of Physical Chemistry, Vol 61, Leone, S. R.; Cremer, P. S.; Groves, J. T.; Johnson, M. A.; Richmond, G., Eds. 2010; Vol. 61, pp 149-170.

29. Karman, T.; Besemer, M.; van der Avoird, A.; Groenenboom, G. C., Diabatic states, nonadiabatic coupling, and the counterpoise procedure for weakly interacting open-shell molecules. J. Chem. Phys. 2018, $148(9), 094105$.

30. Li, S. L.; Truhlar, D. G.; Schmidt, M. W.; Gordon, M. S., Model space diabatization for quantum photochemistry. J. Chem. Phys. 2015, 142 (6), 064106.

31. Wittenbrink, N.; Venghaus, F.; Williams, D.; Eisfeld, W., A new approach for the development of diabatic potential energy surfaces: Hybrid block-diagonalization and diabatization by ansatz. J. Chem. Phys. 2016, 145 (18), 184108.

32. Subotnik, J. E.; Alguire, E. C.; Ou, Q.; Landry, B. R.; Fatehi, S., The requisite electronic structure theory to describe photoexcited nonadiabatic dynamics: nonadiabatic derivative couplings and diabatic electronic couplings. Acc. Chem. Res. 2015, 48 (5), 1340-1350. 
33. Xie, Y.; Jiang, S.; Zheng, J.; Lan, Z., Construction of Vibronic Diabatic Hamiltonian for ExcitedState Electron and Energy Transfer Processes. J. Phys. Chem. A 2017, 121 (50), 9567-9578.

34. Kolomeisky, A. B.; Feng, X. T.; Krylov, A. I., A Simple Kinetic Model for Singlet Fission: A Role of Electronic and Entropic Contributions to Macroscopic Rates. J. Phys. Chem. C 2014, 118 (10), $5188-5195$.

35. Crespo-Otero, R.; Barbatti, M., Recent Advances and Perspectives on Nonadiabatic Mixed Quantum-Classical Dynamics. Chem. Rev. 2018, 118 (15), 7026-7068.

36. Lischka, H.; Nachtigallova, D.; Aquino, A. J. A.; Szalay, P. G.; Plasser, F.; Machado, F. B. C.; Barbatti, M., Multireference Approaches for Excited States of Molecules. Chem. Rev. 2018, 118 (15), 7293-7361.

37. Morrison, A. F.; Herbert, J. M., Analytic derivative couplings and first-principles exciton/phonon coupling constants for an ab initio Frenkel-Davydov exciton model: Theory, implementation, and application to compute triplet exciton mobility parameters for crystalline tetracene. J. Chem. Phys. 2017, $146(22), 224110$.

38. Morrison, A. F.; Herbert, J. M., Evidence for Singlet Fission Driven by Vibronic Coherence in Crystalline Tetracene. J. Phys. Chem. Lett. 2017, 8 (7), 1442-1448.

39. Skourtis, S. S.; Liu, C. R.; Antoniou, P.; Virshup, A. M.; Beratan, D. N., Dexter energy transfer pathways. Proc. Natl. Acad. Sci. U.S.A. 2016, 113 (29), 8115-8120.

40. Dexter, D. L., A Theory of Sensitized Luminescence in Solids. J. Chem. Phys. 1953, $21,836$.

41. Tang, J., Resonance effects on superexchange and sequential electron-transfer reactions due to energy-level crossing. Chem. Phys. 1994, 189 (3), 427-442.

42. Troisi, A.; Orlandi, G., Hole Migration in DNA: a Theoretical Analysis of the Role of Structural Fluctuations. J. Phys. Chem. B 2002, 106 (8), 2093-2101. 
43. Gajdos, F.; Valner, S.; Hoffmann, F.; Spencer, J.; Breuer, M.; Kubas, A.; Dupuis, M.; Blumberger, J., Ultrafast Estimation of Electronic Couplings for Electron Transfer between pi-Conjugated Organic Molecules. J. Chem. Theory Comput. 2014, 10 (10), 4653-4660.

44. Hoffmann, R., An Extended Hückel Theory. I. Hydrocarbons. J. Chem. Phys. 1963, 39 (6), $1397-$ 1412.

45. Cheung, D. L.; Troisi, A., Theoretical Study of the Organic Photovoltaic Electron Acceptor PCBM: Morphology, Electronic Structure, and Charge Localization. J. Phys. Chem. C 2010, 114 (48), 20479-20488.

46. Feng, X. T.; Krylov, A. I., On couplings and excimers: lessons from studies of singlet fission in covalently linked tetracene dimers. Phys. Chem. Chem. Phys. 2016, 18 (11), 7751-7761.

47. Chan, W. L.; Berkelbach, T. C.; Provorse, M. R.; Monahan, N. R.; Tritsch, J. R.; Hybertsen, M. S.; Reichman, D. R.; Gao, J. L.; Zhu, X. Y., The Quantum Coherent Mechanism for Singlet Fission: Experiment and Theory. Acc. Chem. Res. 2013, 46 (6), 1321-1329.

48. Mirjani, F.; Renaud, N.; Gorczak, N.; Grozema, F. C., Theoretical Investigation of Singlet Fission in Molecular Dimers: The Role of Charge Transfer States and Quantum Interference. J. Phys. Chem. C 2014, 118 (26), 14192-14199.

49. Reed, A. E.; Curtiss, L. A.; Weinhold, F., Intermolecular interactions from a natural bond orbital, donor-acceptor viewpoint. Chem. Rev. 1988, 88 (6), 899-926.

50. Schmidt, R., Quantitative determination of ${ }^{1} \Sigma_{\mathrm{g}}{ }^{+}$and ${ }^{1} \Delta_{\mathrm{g}}$ singlet oxygen in solvents very different polarity. General energy gap law for rate constants of electronic energy transfer to and from $\mathrm{O}_{2}$ in the absence of charge-transfer interactions. J. Phys. Chem. A 2006, 110 (34), 10369-10369.

51. Paterson, M. J.; Christiansen, O.; Jensen, F.; Ogilby, P. R., Invited review - Overview of theoretical and computational methods applied to the oxygen-organic molecule photosystem. Photochem. Photobiol. 2006, 82 (5), 1136-1160. 
52. Scurlock, R. D.; Wang, B. J.; Ogilby, P. R., Chemical reactivity of singlet sigma oxygen $\left(\mathrm{b}^{1} \sum_{\mathrm{g}}{ }^{+}\right)$ in solution. J. Am. Chem. Soc. 1996, 118 (2), 388-392.

53. Bodesheim, M.; Schmidt, R., Chemical reactivity of sigma singlet oxygen $\mathrm{O}_{2}\left({ }^{1} \Sigma_{\mathrm{g}}{ }^{+}\right)$. J. Phys. Chem. A 1997, 101 (31), 5672-5677.

54. Bodesheim, M.; Schutz, M.; Schmidt, R., Triplet-state Energy-dependence of the Competitive Formation of $\mathrm{O}_{2}\left({ }^{1} \Sigma_{\mathrm{g}}{ }^{+}\right), \mathrm{O}_{2}\left({ }^{1} \Delta_{\mathrm{g}}\right)$ and $\mathrm{O}_{2}\left({ }^{3} \Sigma_{\mathrm{g}}{ }^{-}\right)$In the Sensitization of $\mathrm{O}_{2}$ by Triplet-states. Chem. Phys. Lett. 1994, $221(1-2), 7-14$.

55. Chou, P. T.; Wei, G. T.; Lin, C. H.; Wei, C. Y.; Chang, C. H., Direct spectroscopic evidence of photosensitized $\mathrm{O}_{2} 765 \mathrm{~nm}\left({ }^{1} \Sigma_{\mathrm{g}}{ }^{+} \rightarrow{ }^{3} \Sigma_{\mathrm{g}}{ }^{-}\right)$and $\mathrm{O}_{2}$ dimol 634 and $703 \mathrm{~nm}\left(\left({ }^{1} \Delta_{\mathrm{g}}\right)_{2} \rightarrow\left({ }^{3} \Sigma_{\mathrm{g}}{ }^{-}\right)_{2}\right)$ vibronic emission in solution. J. Am. Chem. Soc. 1996, 118 (12), 3031-3032.

56. Ogilby, P. R., Singlet oxygen: there is indeed something new under the sun. Chem. Soc. Rev. 2010, $39(8), 3181-3209$.

57. Kearns, D. R., Physical and chemical properties of singlet molecular oxygen. Chem. Rev. 1971, $71(4), 395-427$. 
TOC graph:

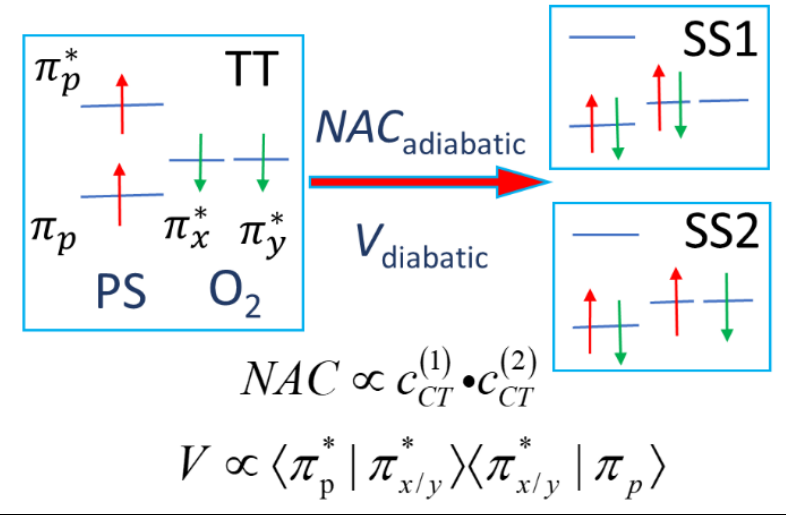

